\title{
Asymptotic Solutions to the Knizhnik-Zamolodchikov Equation and Crystal Base
}

\author{
A.N. Varchenko \\ Department of Mathematics, University of North Carolina, Chapel Hill, NC 27599, USA \\ email address: varchenko@math.unc.edu
}

Received: 14 April 1994/in revised form: 21 October 1994

Dedicated to the memory of Ansgar Schnizer

\begin{abstract}
The Knizhnik-Zamolodchikov equation associated with $s l_{2}$ is considered. The transition functions between asymptotic solutions to the KnizhnikZamolodchikov equation are described. A connection between asymptotic solutions and the crystal base in the tensor product of modules over the quantum group $U_{q} s l_{2}$ is established, in particular, a correspondence between the Bethe vectors of the Gaudin model of an inhomogeneous magnetic chain and the $\mathbb{Q}$-basis of the crystal base.
\end{abstract}

\section{Introduction}

In this work we describe transition functions between asymptotic solutions to the Knizhnik-Zamolodchikov (KZ) equation and establish a connection between asymptotic solutions and the crystal base in the tensor product of modules over a quantum group.

We consider the $\mathrm{KZ}$ equation associated with $s l_{2}$ and the quantum group $U_{q} s l_{2}$, general case can be considered similarly.

For a positive integer $m$, denote by $L(m)$ the $s l_{2}$ irreducible module with highest weight $m$. For positive integers $m_{1}, \ldots, m_{n}$, set $L=L\left(m_{1}\right) \otimes \cdots \otimes L\left(m_{n}\right)$.

Let $\Omega=\frac{1}{2} h \otimes h+e \otimes f+f \otimes e \in s l_{2}^{\otimes 2}$ be the Casimir operator. For $i \neq j$ denote by $\Omega_{i j}$ the linear operator on $L$ which acts as $\Omega$ on the $i^{\text {th }}$ and $j^{\text {th }}$ factors and as the identity on the other factors. The $\mathrm{KZ}$ equation on an $L$-valued function $\psi\left(z_{1}, \ldots, z_{n}\right)$ is the system of equations

$$
\frac{\partial \psi}{\partial z_{j}}=\frac{1}{\kappa} \sum_{l \neq l} \frac{\Omega_{j l}}{z_{j}-z_{l}} \psi, \quad j=1, \ldots, n,
$$

where $\kappa$ is a complex parameter. In this paper we assume that $\kappa$ is not a rational number. The $\mathrm{KZ}$ equation is defined over $\mathscr{U}_{n}=\left\{z \in \mathbb{C}^{n} \mid z_{i} \neq z_{j}\right.$ for $\left.i \neq j\right\}$. 
For any permutation $\sigma \in S_{n}$, define an open convex unbounded polytope

$$
D_{\sigma}=\left\{z \in \mathbb{R}^{n} \mid z_{\sigma(1)}<\cdots<z_{\sigma(n)}\right\} .
$$

One can easily resolve singularities of $D_{\sigma}$ and cover $D_{\sigma}$ by local charts with local coordinates $u_{1}, \ldots, u_{n}$ such that:

$$
u_{n}=z_{1}+\cdots+z_{n},
$$

$D_{\sigma}$ is defined in this chart by inequalities $u_{1}>0, \ldots, u_{n-1}>0$,

The $\mathrm{KZ}$ equation in this chart has the form

$$
\frac{\partial \psi}{\partial u_{n}}=0, \frac{\partial \psi}{\partial u_{j}}=\frac{1}{\kappa} H_{j}, \quad j=1, \ldots, 1-n,
$$

where $H_{J}=\Omega_{j} / u_{j}+\operatorname{Reg}_{j}, \Omega_{j}$ is a constant operator on $L$, and $\operatorname{Reg}$, is an operator regular at $u=0$.

We call such charts asymptotic zones; they are numerated by suitable trees $T$.

The operators $\Omega_{1}, \ldots, \Omega_{n-1}$ commute and have a common eigenbasis. We distinguish an eigenbasis $\mathscr{B}=\left(v_{1}(\kappa), \ldots, v_{N}(\kappa)\right)$ for nonrational $\kappa$. For every $j$ the vector $v_{j}(\kappa)$ is proportional to a fixed common eigenvector of $\Omega_{1}, \ldots, \Omega_{n-1}$, and the coefficient of the proportionality appropriately depends on $\kappa$, see (2.3.1).

For each $j=1, \ldots, N$ and a nonrational $\kappa$, there exists a unique solution $\psi_{J}$ to the $\mathrm{KZ}$ equation with parameter $\kappa$ defined in $D_{\sigma}$ and such that

$$
\psi_{j}=\left(v_{j}(\kappa)+\mathcal{O}(u, k)\right) \prod_{l=1}^{n-1} u_{l}^{\mu_{l} / \kappa}
$$

where $\mu_{l}$ is the eigenvalue of $\Omega_{l}$ at $v_{j}, \mathcal{O}(u, \kappa)$ is a regular function of $u$ at $u=0$, and $\mathcal{O}(0, \kappa)=0$. (Notice that all numbers $\mu_{l}$ are real.)

Thus, for each asymptotic zone $(T, \sigma)$ we construct a fundamental system of solutions $\psi_{T, \sigma}=\left\{\psi_{j}\right\}$.

Our first main result gives explicit transition functions between these fundamental solutions $\psi_{T, \sigma}$ and $\psi_{T^{\prime}}, \sigma^{\prime}$ in terms of the $q$-6j-symbols, where $q=q(\kappa)$ and $q(\kappa)=\exp (2 \pi i / \kappa)$, see $(2.4)$.

In particular, if $\kappa=i s, s \in \mathbb{R}, s \rightarrow+0$, then $q(\kappa) \rightarrow \infty$, and our formulae show that for every $\sigma, T, T^{\prime}$ the transition function between the fundamental systems of solutions $\psi_{T, \sigma}$ and $\psi_{T^{\prime}, \sigma}$ has the form $\mathbf{1}+\mathcal{O}\left(q(\kappa)^{-\frac{1}{4}}\right)$, where $\mathbf{1}$ is the unit matrix and the solutions composing $\psi_{T, \sigma}$ and $\psi_{T^{\prime}, \sigma}$ must be suitably enumerated, see (2.4) and (1.3.12).

Assume that $\kappa=i s, s \in \mathbb{R}$ and $s \rightarrow+0$. For any asymptotic zone consider the fundamental system of solutions defined by (0.4). Our second main result, see (2.5), states that for every $j$ the solution $\psi_{J}$ has an asymptotic expansion of the form

$$
\psi_{J}=\prod_{l=1}^{n-1} u_{l}^{-\imath \mu_{l} / s} \exp (-i S / s) \sum_{l=0}^{\infty} f_{l} s^{l}
$$

where $S, f_{l}$ are real-analytic functions of $u$ in our chart regular at $u=0$, the function $S$ is real valued, the functions $f_{l}$ are $L$-valued, and, in particular,

$$
\left|\prod_{l=1}^{n-1} u_{l}^{-i \mu_{l} / s} \exp (-i S / s)\right|=1 \text {. }
$$


The first term $f_{0}$ is a common eigenvector of the operators $H_{1}, \ldots, H_{n-1}$. The first terms of asymptotics of the solutions $\psi_{J}$ form an eigenbasis of the operators $H_{1}, \ldots, H_{n-1}$. We describe its limit at $u \rightarrow 0$.

If the $\mathrm{KZ}$ equation has an asymptotic solution of the form

$$
\psi(u)=\exp (P(u) / \kappa) \sum_{l=0}^{\infty} g_{l}(u) \cdot \kappa^{l}
$$

one can show that $g_{0}(u)$ must be a common eigenvector of $H_{1}, \ldots, H_{n-1}$ and the function $g_{0}(u)$ is determined up to multiplication by a constant [RV]. One can deduce from this remark that a solution having an asymptotic expansion of the form described in (0.5) is unique up to multiplication by a function of $s$ which has the form $1+\mathcal{O}(s)$, where $\mathcal{O}(0)=0$.

Our two main results have the following quantum group interpretation.

Let $U_{q}$ be the quantum group corresponding to the $s l_{2}$ and $U_{q=q(\kappa)}$ its specialization at $q=q(\kappa)$. For a positive integer $m$ denote by $L(m, q)$ (resp. $L(m, q=$ $q(\kappa))$ ) the irreducible $U_{q}$ (resp. $U_{q=q(\kappa)}$ ) module with highest weight $m$. For positive integers $m_{1}, \ldots, m_{n}$ and a permutation $\sigma$ denote by $L^{\sigma}(q)$ the product $L\left(m_{\sigma(1)}, q\right) \otimes \cdots \otimes L\left(m_{\sigma(n)}, q\right)$. Denote by $L^{\sigma}(q=q(\kappa))$ the corresponding product for $U_{q=q(\kappa)}$. Denote by $\operatorname{Sol}(\kappa)_{\sigma}$ the space of solutions over $D_{\sigma}$ to the $\mathrm{KZ}$ equation with parameter $\kappa$.

Our formulae for transition functions between the constructed fundamental system of solutions allows us to construct an isomorphism

$$
\pi_{\sigma}(\kappa): L^{\sigma}(q=q(\kappa)) \cong \operatorname{Sol}(\kappa)_{\sigma}
$$

and show, once again, that the monodromy representation of the $\mathrm{KZ}$ equation is isomorphic to the $R$-matrix representation of the braid group, where $R$ is the universal $R$-matrix of $U_{q=q(\kappa)}$, see [K, Dr, V1, FW]. Under the isomorphism $\pi_{\sigma}(\kappa)$ each of the constructed fundamental systems of solutions $\psi_{T, \sigma}$ gives a basis $\mathscr{B}_{T, \sigma}(\kappa)$ in $L^{\sigma}(q=q(\kappa))$, see $(2.4)$.

In [Ka] the notion of a crystal base is defined. The module $L(m, q)$ has a standard crystal base when $q \rightarrow \infty$. The tensor product of the standard crystal bases gives a distinguished crystal base in $L^{\sigma}(q)$. Our isomorphism $\pi_{\sigma}(\kappa)$ has the following property:

For every $T$, the fundamental system of solutions $\psi_{T, \sigma}$ lifts to the same distinguished crystal base of $L^{\sigma}(q)$, see (2.6).

Therefore, we may conclude that the distinguished crystal base is given by $(0.4)$ and normalized by property $(0.5)$. This gives an "asymptotic" definition of the crystal base.

The proof of transition formulae between the fundamental solutions and the proof of property $(0.5)$ are based on integral representations for solutions to the $\mathrm{KZ}$ equation, see [SV, V1]. Any solution in $\operatorname{Sol}(\kappa)_{\sigma}$ can be represented as a linear combination of solutions of the form

$$
\psi(z)=\int_{\gamma(z)} \Phi M(t, z)
$$


where $M$ is a rational $L$-valued differential form of $z$ and some variable $t=$ $\left(t_{1}, \ldots, t_{k}\right), \gamma(z)$ is a family of cycles in $t$-space,

$$
\Phi(t, z)=\prod_{1 \leqq l<j \leqq n}\left(z_{j}-z_{l}\right)^{m_{l} m_{j} / 2 \kappa} \prod_{1 \leqq l<j \leqq k}\left(t_{l}-t_{J}\right)^{2 / \kappa} \prod_{l=1}^{k} \prod_{j=1}^{n}\left(z_{j}-t_{l}\right)^{-m_{J} / \kappa} .
$$

In this construction cycles $\gamma(z)$ numerate solutions to the $\mathrm{KZ}$ equation. In [V1] we identified the homology group of such cycles for a fixed $k$ with the subspace of singular vectors in $L^{\sigma}(q=q(\kappa))$ of a suitable weight, see precise statements in [V1]. This construction after suitable renormalization gives us the isomorphism $\pi_{\sigma}$, see (4.4).

If $\kappa=i s, s \rightarrow+0$, then integral (0.6) is localized at critical points of the function $\Phi$ with respect to the variable $t$, and one can compute asymptotics of the solution applying the method of steepest descent, see [RV, V2]. One can push the cycle $\gamma(z)$ onto $t$-critical points of $\Phi$. If $\gamma(z)$ "sits" on some of the $t$-critical points then each of the points gives its input into the asymptotic expansion of the solution.

The symmetric group $S_{k}$ of permutations of coordinates $t_{1}, \ldots, t_{k}$ acts on the set of critical points of $\Phi$.

In each asymptotic zone $(T, \sigma)$, one can describe asymptotics of $t$-critical points of $\Phi$. It turns out that the $S_{k}$ orbits of $t$-critical points of $\Phi$ are in correspondence with solutions $\psi_{J}$ composing the fundamental system $\psi_{T, \sigma}$. For any $j$ we distinguish a cycle $\gamma_{J}(z)$ such that

(1) $\psi_{j}=\int_{\gamma_{j}(z)} \Phi M$,

(2) $\gamma_{j}(z)$ sits exactly on one $S_{k}$ orbit of $t$-critical points,

(3) the asymptotic expansion of the integral at this orbit has property (0.5).

This gives a "topological" definition of the crystal base as the base in the homology group of cycles corresponding to a fixed $z$. This base is formed by the classes numerated by the orbits of $t$-critical points and each such a class has a representing chain sitting on one orbit. In this construction $z$ must be in an asymptotic zone.

The value of the function $M$ at a $t$-critical point $(t(z), z)$ of the function $\Phi$ gives the first term of asymptotics as $k=i s$ and $s \rightarrow+0$. This value $M(t(z), z)$ is an eigenvector of the commuting operators $H_{1}(z), \ldots, H_{n-1}(z)$.

The algebraic Bethe Ansatz is a construction of eigenvectors for a system of commuting operators. One considers a vector valued function of a special form and determines its arguments in such a way that the value of this function is an eigenvector. The equations which determine these special values of arguments are called the Bethe equations. The eigenvector is called a Bethe vector, for more details see [FT].

One of the systems of commuting operators which can be diagonalized by the ABA is the Gaudin model of an inhomogeneous magnetic chain [G].

It turns out that the function $M(t, z)$ is exactly the special function which appear in the $\mathrm{ABA}$ for the Gaudin model and the Bethe equations for the Gaudin model coincide with the equations on $t$-critical points of the function $\Phi$, see [B, BF, RV].

This relation to the $\mathrm{ABA}$ and the above formulated results give us a correspondence between the $\mathbb{Q}$-basis of the distinguished crystal base and the Bethe vectors of the Gaudin model, see (2.6.5), or, more generally, between the crystallization phenomenon in the theory of quantum groups and the Bethe ansatz construction in statistical mechanics. On the Bethe ansatz and the $\mathrm{KZ}$ equation see also [FFR]. 
Section 1 contains preliminary information on bases in $L$ and $L^{\sigma}(q)$. In Sect. 2 we state the formulae for transition functions and the theorem about asymptotic expansion (0.5). In Sect. 3 we review integral representations for solutions and describe asymptotics of critical points and the corresponding Bethe vectors. Section 4 contains the homological part of the work.

\section{Bases in Tensor Products}

(1.1). Quantum Group $U_{q} s l_{2}$. The $\mathbb{Q}\left(q^{1 / 4}\right)$-algebra $U_{q}=U_{q} s l_{2}$ is the algebra generated by the symbols $e, f, h$ with the relations

$$
\begin{aligned}
& {[h, e]=2 e,} \\
& {[h, f]=-2 f,} \\
& {[e, f]=q^{h / 2}-q^{-h / 2} .}
\end{aligned}
$$

Here $q^{1 / 4}$ is an indeterminate.

Remark. Usually one considers $U_{q}$ as an $\mathbb{Q}(q)$ algebra. Our choice of $q^{1 / 4}$ instead of $q$ is motivated by topological considerations in Sect. 4 .

$U_{q}$ has a Hopf algebra structure with the comultiplication $\Delta$ defined by

$$
\begin{aligned}
& \Delta(h)=h \otimes 1+1 \otimes h, \\
& \Delta(e)=e \otimes q^{h / 2}+1 \otimes e, \\
& \Delta(f)=f \otimes 1+q^{-h / 2} \otimes f .
\end{aligned}
$$

By $\Delta$, the tensor product of $U_{q}$-modules has a structure of $U_{q}$-module.

Let

$$
\begin{aligned}
{[n]_{q} } & =\left(q^{n / 2}-q^{-n / n}\right) /\left(q^{1 / 2}-q^{-1 / 2}\right), \\
(n)_{q} & =q^{n / 2}-q^{-n / 2}, \\
{[n]_{q} ! } & =\prod_{k=1}^{n}[k]_{q}, \\
e^{(n)} & =\frac{e^{n}}{[n]_{q} !}, \quad e^{(0)}=1, \\
f^{(n)} & =\frac{f^{n}}{[n]_{q} !}, \quad f^{(0)}=1 .
\end{aligned}
$$

Let $V_{1}$ and $V_{2}$ be $U_{q}$-modules. The modules $V_{1} \otimes V_{2}$ and $V_{2} \otimes V_{1}$ are isomorphic. The isomorphism is given by the formula

$$
V_{1} \otimes V_{2} \stackrel{R}{\rightarrow} V_{1} \otimes V_{2} \stackrel{P}{\rightarrow} V_{2} \otimes V_{1}
$$

where $P$ is the transposition of the factors. $R \in U_{q} \widehat{\otimes} U_{q}$ is the universal $R$-matrix, and $\widehat{\otimes}$ denotes a proper completion of the tensor product. Denote the isomorphism $P R$ by $\hat{R}$. 
Denote by $S_{n}$ the group of permutations of the set $\{1, \ldots, n\}$, by $B_{n}$ the braid group on $n$ strings with the standard generators $\sigma_{1}, \ldots, \sigma_{n-1}$, by $\tau: B_{n} \rightarrow S_{n}$, the natural epimorphism.

Let $V_{1}, \ldots, V_{n}$ be $U_{q}$-modules. For $j=1, \ldots, n-1$, define an $U_{q}$-isomorphism.

$$
R_{j, j+1}: V_{1} \otimes \cdots \otimes V_{n} \rightarrow V_{1} \otimes \cdots \otimes V_{j+1} \otimes V_{j} \otimes \cdots \otimes V_{n},
$$

as the map which acts as $\hat{R}$ on $V_{j} \otimes V_{j+1}$ and as the identity on the other factors.

For any braid $\sigma \in B_{n}$ these isomorphisms induce a well defined isomorphism

$$
R_{\sigma}: V_{1} \otimes \cdots \otimes V_{n} \rightarrow V_{\tau \sigma(1)} \otimes \cdots \otimes V_{\tau \sigma(n)} .
$$

(1.2). Bases in Tensor Products. For $m \in \mathbb{Z}$ let $V(m)$ be the Verma module with highest weight $m . V(m)$ is generated by its singular vector $v_{m}$ such that $e v_{m}=0$ and $q^{h} v_{m}=q^{m} v_{m}$. The elements $f^{(j)} v_{m}, j \geqq 0$, form a basis of $V(m)$. For a nonnegative $m$ the vector $f^{(m+1)} v_{m}$ generates a proper submodule of $V(m)$. The quotient, $L(m)$, is the $(m+1)$-dimensional irreducible $U_{q}$-module with a basis generated by $v_{m}, f^{(1)} v_{m}, \ldots, f^{(m)} v_{m}$. ules:

The tensor product of irreducible modules is the direct sum of irreducible mod-

$$
L\left(m_{1}\right) \otimes L\left(m_{2}\right)=L\left(\left|m_{1}-m_{2}\right|\right) \oplus L\left(\left|m_{1}-m_{2}\right|+2\right) \oplus \cdots \oplus L\left(m_{1}+m_{2}\right),
$$

and a singular vector of $L\left(m_{1}+m_{2}-2 k\right)$ has the form

$$
\begin{aligned}
\left(v_{m_{1}}, k v_{m_{2}}\right)= & \sum_{p=0}^{k}(-1)^{p} \frac{\left(m_{2}-k+1\right)_{q} \cdots\left(m_{2}-k+p\right)_{q}}{\left(m_{1}\right)_{q} \cdots\left(m_{1}-p+1\right)_{q}} \\
& \cdot q^{-p\left(m_{2}-2 k+p+1\right) / 2} f^{(p)} v_{m_{1}} \otimes f^{(k-p)} v_{m_{2}} .
\end{aligned}
$$

This decomposition gives a basis $\left\{f^{(l)}\left(v_{m_{1}}, k_{k} v_{m_{2}}\right)\right\}$ in the tensor product.

The $U_{q}$-isomorphism

$$
\hat{R}: L\left(m_{1}\right) \otimes L\left(m_{2}\right) \rightarrow L\left(m_{2}\right) \otimes L\left(m_{1}\right)
$$

is given by

$$
\begin{gathered}
f^{(l)}\left(v_{m_{1}},{ }_{k} v_{m_{2}}\right) \mapsto R\left(m_{1}, m_{2} ; k\right)_{q} f^{l}\left(v_{\left.m_{2},{ }_{k} v_{m_{1}}\right),}\right. \\
R\left(m_{1}, m_{2} ; k\right)_{q}=(-1)^{k} q^{\left(m_{1} m_{2}-4 k m_{2}+2 k(k+1)\right) / 4} \frac{\left(m_{2}\right)_{q} \cdots\left(m_{2}-k+1\right)_{q}}{\left(m_{1}\right)_{q} \cdots\left(m_{1}-k+1\right)_{q}} .
\end{gathered}
$$

There are two ways to decompose $L\left(m_{1}\right) \otimes L\left(m_{2}\right) \otimes L\left(m_{3}\right)$ into irreducibles. These two ways give two bases in the triple tensor product: $\left\{f^{(l)}\left(\left(v_{m_{1}}, k_{1} v_{m_{2}}\right), k_{2} v_{m_{3}}\right)\right\}$ and $\left\{f^{(l)}\left(v_{m_{1}}, k_{1}\left(v_{m_{2}}, k_{2} v_{m_{3}}\right)\right)\right\}$.

The matrix elements of the matrix, connecting these bases, are called the $6 j$ symbols:

$$
\begin{aligned}
& f^{(l)}\left(\left(v_{m_{1}}, k_{1} v_{m_{2}}\right), k_{2} v_{m_{3}}\right) \\
& =\sum_{k}\left\{\begin{array}{ll}
m_{1}, & m_{2}, m_{3} \\
k_{1}, & k_{2}, k
\end{array}\right\}_{q} f^{(l)}\left(v_{m_{1}}, k\left(v_{m_{2}}, k_{1}+k_{2}-k v_{m_{3}}\right)\right) .
\end{aligned}
$$


There are numerous formulae for $6 j$-symbols, see, for example, [KR].

The singular vectors $\left(v_{m_{1}}, k v_{m_{2}}\right),\left(\left(v_{m_{1}}, k_{1} v_{m_{2}}\right), k_{2} v_{m_{3}}\right),\left(v_{m_{1}}, k_{1}\left(v_{m_{2}}, k_{2} v_{m_{3}}\right)\right)$, and the matrix of $6 j$-symbols can be visualized as follows.
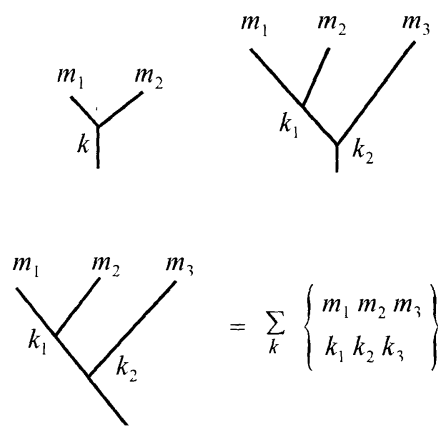

$$
=\sum_{k}\left\{\begin{array}{l}
m_{1} m_{2} m_{3} \\
k_{1} k_{2} k_{3}
\end{array}\right\}
$$
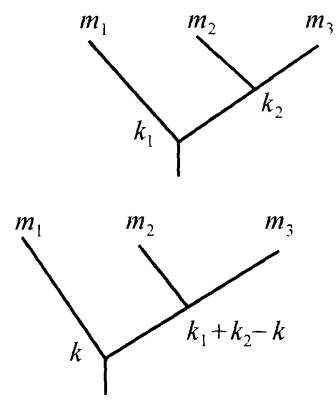

The braid group $B_{3}$ acts on the triple tensor products. The basis $\left(\left(v_{m_{1}}, k_{1} v_{m_{2}}\right), k_{2} v_{m_{3}}\right)$ is an eigenbasis for $R_{1,2}$, and the basis $\left(v_{m_{1}}, k_{1}\left(v_{m_{2}}, k_{2} v_{m_{2}}\right)\right)$ is an eigenbasis for $R_{2,3}$.
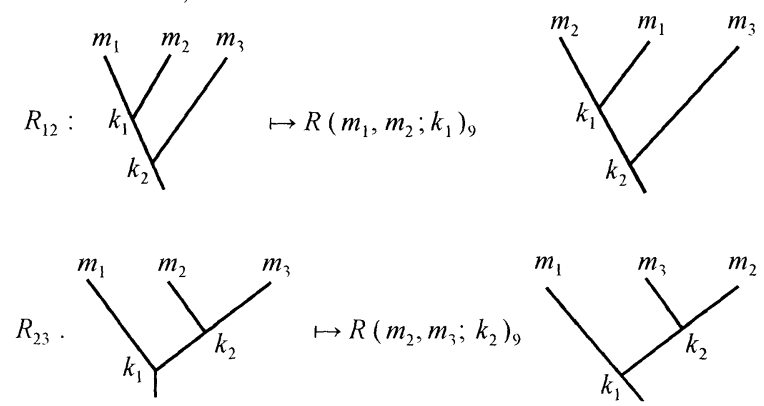

Consider the tensor product of $n$ irreducible modules. We describe its bases, the connecting matrices, and the $R$-matrix action.

An $n$-tree is a planar tree with $n$ tops, one root, and $(n-1)$ internal triple vertices. We numerate the tops by indices $1, \ldots, n$ from left to right.

See an example in (1.2.7):

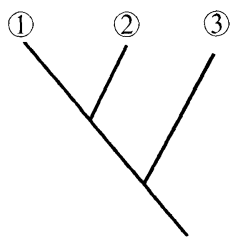

For an $n$-tree we denote the set of its internal vertices by $\operatorname{In}_{T}$. We say that an $n$-tree has marked tops if a nonnegative integer is assigned to every top. Denote by $m_{j}$ the number assigned to the $j^{\text {th }}$ top.

For an $n$-tree $T$ with marked tops, we construct a basis in $L\left(m_{1}\right) \otimes \cdots \otimes L\left(m_{n}\right)$, denoted by $\mathscr{B}_{T}$.

$A$ coloring of $T$ is a map $c: \operatorname{In}_{T} \rightarrow \mathbb{Z}_{\geqq 0}$. Define the weight of a coloring as the number

$$
m(c)=m_{1}+\cdots+m_{n}-2 \sum_{w \in \operatorname{In}_{T}} c(w) .
$$


Delete an internal vertex $w$, then the tree is decomposed into three trees: the left branch, the right branch, and the part containing the root.

Denote by $J^{l e}(w)$ the set of indices of the tops lying in the left branch of $w$, denote by $I^{l e}(w)$ the set of the internal vertices lying in the left branch of $w$. For a coloring $c$, define the weight of the left branch as the number

$$
m^{l e}(w)=\sum_{j \in J^{l e}} m_{j}-2 \sum_{u \in I^{l e}} c(u) .
$$

Let $J^{r}(w), I^{r}(w)$, and $m^{r}(w)$ be the sets and the weight defined for the right branch analogously.

Say that a coloring $c$ is admissible at a vertex $w$ if $c(w) \leqq \min \left(m^{l e}(w), m^{r}(w)\right)$. Say that a coloring of an $n$-tree $T$ with marked tops is admissible it is admissible at every internal vertex.

The elements of the basis $\mathscr{B}_{T}$ are numerated by the pairs $(c, l)$, where $c$ is an admissible coloring and $l=0, \ldots, m(c)$. The vector corresponding to $(c, l)$ is defined inductively on $n$. Namely, let $w$ be the internal vertex of $T$ which is neighboring to the root of $T$. Let $T^{l e}$ and $T^{r}$ be the left and right branches of $w$. The coloring $c$ induces colorings $c^{l e}$ and $c^{r}$ of $T^{l e}$ and $T^{r}$, respectively. Let $1, \ldots, i$ be the indices of the tops lying in the left branch of $w$. Let $v^{l e}$ be the vector in $L\left(m_{1}\right) \otimes \cdots \otimes$ $L\left(m_{i}\right)$ corresponding to the pair $\left(c^{l e}, 0\right)$ and $v^{r}$ the vector in $L\left(m_{l+1}\right) \otimes \cdots \otimes L\left(m_{n}\right)$ corresponding to $\left(c^{r}, 0\right)$. If $i=1$, then $v^{l e}=v_{m_{1}}$, where $v_{m_{1}}$ is the singular vector of $L\left(m_{1}\right)$. If $i=n-1$, then $v^{r}=v_{m_{n}}$, where $v_{m_{n}}$ is the singular vector of $L\left(m_{n}\right)$. Define the vector $v(c, l, T)$ by

$$
v(c, l, T)=f^{(l)}\left(v^{l e},{ }_{c(w)} v^{r}\right),
$$

where $(\cdot, c(w) \cdot)$ is defined by (1.2.1).

The $U_{q}$-action is given by

$$
\begin{aligned}
q^{h} v(c, l, T) & =q^{m(c)-2 l} v(c, l, T), \\
e v(c, l, T) & =(m(c)-l+1)_{q} v(c, l-1, T) \text { for } l>0, \\
e v(c, 0, T) & =0, \\
f v(c, l, T) & =[l+1]_{q} v(c, l+1, T) \text { for } l<m(c), \\
f v(c, m(c), T) & =0 .
\end{aligned}
$$

Let $T$ and $T^{\prime}$ be $n$-trees. Let $w_{1}$ and $w_{2}$ be internal vertices of $T$ and $w_{1}^{\prime}$ and $w_{2}^{\prime}$ internal vertices of $T^{\prime}$. Say that $T$ and $T^{\prime}$ are adjacent at $w_{1}, w_{2}, w_{1}^{\prime}, w_{2}^{\prime}$, if in a neighborhood of the vertices the trees have the form shown in (1.2.11), and the trees are identical outside the neighborhood.
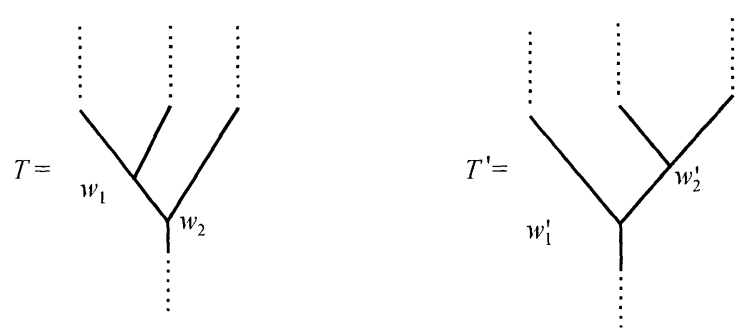
Let $T$ and $T^{\prime}$ be adjacent. Let $c$ and $c^{\prime}$ be admissible colorings of $T$ and $T^{\prime}$ respectively. Say that the colorings are similar, $c \equiv c^{\prime}$, if the colorings are equal at the corresponding internal vertices of $T$ and $T^{\prime}$ different from $w_{1}, w_{2}, w_{1}^{\prime}, w_{2}^{\prime}$, and if $c\left(w_{1}\right)+c\left(w_{2}\right)=c^{\prime}\left(w_{1}^{\prime}\right)+c^{\prime}\left(w_{2}^{\prime}\right)$.

The matrix, connecting the bases $\mathscr{B}_{T}$ and $\mathscr{B}_{T^{\prime}}$ of adjacent trees is given by

$$
v(c, l, T)=\sum_{c^{\prime} \equiv c}\left\{\begin{array}{ccc}
m^{l e}\left(w_{1}\right) & m^{r}\left(w_{1}\right) & m^{r}\left(w_{2}\right) \\
c\left(w_{1}\right) & c\left(w_{2}\right) & c^{\prime}\left(w_{1}^{\prime}\right)
\end{array}\right\}_{q} v\left(c^{\prime}, l, T^{\prime}\right)
$$

where \{\}$_{q}$ are $6 j$-symbols.

The matrix connecting the bases $\mathscr{B}_{T}$ and $\mathscr{B}_{T^{\prime}}$ for arbitrary $T$ and $T^{\prime}$ can be written as a product of the matrices connecting adjacent bases.

For $j=1, \ldots, n-1$, we describe the action of the operator

$$
R_{J, j+1}: L\left(m_{1}\right) \otimes \cdots \otimes L\left(m_{n}\right) \rightarrow L\left(m_{1}\right) \otimes \cdots L\left(m_{j+1}\right) \otimes L\left(m_{l}\right) \cdots \otimes L\left(m_{n}\right) .
$$

Denote the first tensor product by $L$ and the second by $L^{\prime}$.

We say that an $n$-tree $T$ is adjacent to the transposition of $j$ and $j+1$ if there exists an internal vertex $w$ such that the $j^{\text {th }}$ top of $T$ forms the left branch of $w$, and the $(j+1)^{\text {th }}$ top of $T$ forms the right branch of $w$. Let $T$ be adjacent to the transposition of $j$ and $j+1$.

Let $\{v(c, l, T ; L)\}$ be the $T$-basis of $L$ and $\left\{v\left(c, l, T ; L^{\prime}\right)\right\}$ the $T$-basis of $L^{\prime}$. Then for every $(c, l)$ we have

$$
R_{J, j+1} v(c, l, T ; L)=R\left(m_{j}, m_{J+1} ; c(w)\right) \cdot v\left(c, l, T ; L^{\prime}\right),
$$

where $R\left(m_{j}, m_{j+1} ; c(w)\right)$ is defined by (1.2.3). As an example see (1.2.3) and (1.2.6).

Formulae (1.2.12) and (1.2.13) describe the action of the braid group $B_{n}$ on the tensor product of $n$ irreducible $U_{q}$-modules.

(1.3). Crystal Base. The tensor product $L\left(m_{1}\right) \otimes \cdots \otimes L\left(m_{n}\right)$ has the trivial basis $f^{\left(l_{1}\right)} v_{m_{1}} \otimes \cdots \otimes f^{\left(l_{n}\right)} v_{m_{n}}, l_{j}=0, \ldots, m_{j}, j=1, \ldots, n$. It turns out that all the tree bases, constructed in (1.2), tend (in an appropriate sense) to the trivial basis as $q \rightarrow \infty$. This phenomenon is described in $[\mathrm{K}]$ and is called crystallization.

For a finite dimensional $U_{q}$-module $M$ and $\lambda \in \mathbb{Z}$, let $M_{\lambda}=\left\{u \in M \mid q^{h} u=q^{\lambda} u\right\}$ be the weight space of weight $\lambda$. We have $M=\bigoplus_{i \in \mathbb{Z}} M_{\lambda}$.

Any element $u$ of $M_{i}$ is uniquely written in the form $u=\sum f^{(l)} u_{l}$, where $u_{l} \in \operatorname{ker} e \cap M_{\lambda+2 l}$. Following [K] define the endomorphisms $\tilde{e}$ and $\tilde{f}$ on $M$ by

$$
\tilde{e} u=\sum f^{(n-1)} u_{n}, \quad \tilde{f} u=\sum f^{(n+1)} u_{n} .
$$

Let $A$ be the subring of $\mathbb{Q}\left(q^{1 / 4}\right)$ consisting of the rational functions of $q^{1 / 4}$ regular at $q^{1 / 4}=\infty$.

$A$ crystal base of $M$ is a pair $(L, B)$ satisfying the following conditions.

$L$ is a free sub- $A$-module of $M$ such that $M=\mathbb{Q}\left(q^{1 / 4}\right) \bigotimes_{A} L$,

$B$ is a base of the $\mathbb{Q}$-vector space $L / q^{-1 / 4} L$,

$$
L=\bigoplus_{\lambda \in \mathbb{Z}} L_{\lambda} \text { and } B=\coprod_{\lambda \in \mathbb{Z}} B_{\lambda} \text {, where }
$$




$$
L_{\lambda}=L \cap M_{\lambda}, \quad B_{\lambda}=B \cap\left(L_{\lambda} / q^{-1 / 4} L_{\lambda}\right),
$$

$\tilde{e} L \subset L$ and $\tilde{f} L \subset L$. Hence $\tilde{e}$ and $\tilde{f}$ operate on $L / q^{-1 / 4} L$,

$\tilde{e} B \subset B \cup\{0\}$ and $\tilde{f} B \subset B \cup\{0\}$,

For $b, b^{\prime} \in B$, we have $b^{\prime}=\tilde{f} b$ if and only if $b=\tilde{e} b^{\prime}$.

For $b \in B$, set $\varepsilon(b)=\max \left\{k \geqq 0 \mid \tilde{e}^{k} b \in B\right\}$ and $\varphi(b)=\max \left\{k \geqq 0 \mid \tilde{f}^{k} b \in B\right\}$. (1.3.7) Example. For $m \in \mathbb{Z}_{\geqq 0}$, consider the irreducible module $L(m)$ with the basis $f^{(l)} v_{m}, l=0, \ldots, m$. We have $\tilde{e} f^{(l)} v_{m}=f^{(l-1)} v_{m}$ and $\tilde{f} f^{(l)} v_{m}=f^{(l+1)} v_{m}$. Let $\mathscr{L}(m)=\bigoplus_{l} A f^{(l)} v_{m}$ and $\mathscr{B}(m)=\left\{f^{(l)} v_{m} \mid l=0, \ldots, m\right\} \subset L / q^{-1 / 4} L$. Then $(\mathscr{L}(m), \mathscr{B}(m))$ is a crystal base of $L(m)$.

(1.3.8) Theorem, [K]. Let $M_{1}$ and $M_{2}$ be two finite dimensional $U_{q}$-modules and let $\left(L_{j}, B_{j}\right)$ be a crystal base of $M_{j}, j=1,2$. Set $L=L_{1} \otimes_{A} L_{2}$ and $B=\left\{b_{1} \otimes b_{2} \in\right.$ $\left.L / q^{-1 / 4} L \mid b_{j} \in B_{j}\right\}$.

(i) Then $(L, B)$ is a crystal base of $M_{1} \otimes M_{2}$.

(ii) For $b_{j} \in B_{j}, j=1,2$, we have

$$
\begin{aligned}
& \tilde{f}\left(b_{1} \otimes b_{2}\right)= \begin{cases}\tilde{f} b_{1} \otimes b_{2} & \text { if } \varphi\left(b_{1}\right)>\varepsilon\left(b_{2}\right) \\
b_{1} \otimes \tilde{f} b_{2} & \text { if } \varphi\left(b_{1}\right) \leqq \varepsilon\left(b_{2}\right),\end{cases} \\
& \tilde{e}\left(b_{1} \otimes b_{2}\right)= \begin{cases}b_{1} \otimes \tilde{e} b_{2} & \text { if } \varphi\left(b_{1}\right)<\varepsilon\left(b_{2}\right) \\
\tilde{e} b_{1} \otimes b_{2} & \text { if } \varphi\left(b_{1}\right) \geqq \varepsilon\left(b_{2}\right) .\end{cases}
\end{aligned}
$$

(1.3.9) Theorem, $[\mathrm{K}]$. Let $(L, B)$ be a crystal base of a finite dimensional $U_{q^{-}}$ module $M$. Then there exists an isomorphism $M \cong \bigoplus_{j} L\left(m_{j}\right)$ by which $(L, B)$ is isomorphic to $\bigoplus_{j}\left(\mathscr{L}\left(m_{j}\right), \mathscr{B}\left(m_{j}\right)\right)$.

Consider the tensor product of $n$ irreducible $U_{q}$-modules, $L=L\left(m_{1}\right) \otimes \cdots \otimes$ $L\left(m_{n}\right)$. Let $\mathscr{L}=\mathscr{L}\left(m_{1}\right) \bigotimes_{A} \cdots \bigotimes_{A} \mathscr{L}\left(m_{n}\right), \mathscr{B}=\mathscr{B}\left(m_{1}\right) \otimes \cdots \otimes \mathscr{B}\left(m_{n}\right)$. By (1.3.8), $(\mathscr{L}, \mathscr{B})$ is a crystal base of $L$. We call $(\mathscr{L}, \mathscr{B})$ the distinguished crystal base of the tensor product $L$.

For any $n$-tree introduce

$$
\begin{aligned}
& \mathscr{L}_{T}=\bigoplus_{(c, l)} A v(c, l, T), \\
& \mathscr{B}_{T}=\{v(c, l, T)\} \subset \mathscr{L}_{T} / q^{-1 / 4} \mathscr{L}_{T} .
\end{aligned}
$$

It follows from $(1.2 .10)$ that $(\mathscr{L}, \mathscr{B})$ is a crystal base of $L$.

(1.3.11) Proposition. For any $n$-tree $T$, we have $(\mathscr{L}, \mathscr{B})=\left(\mathscr{L}_{T}, \mathscr{B}_{T}\right)$.

Proof. It suffices to prove the proposition for $n=2$. Let $\left(v_{m_{1}},{ }_{k} v_{m_{2}}\right)$ be the vector defined by $(1.2 .1)$. Then for any $k=0, \ldots, \min \left(m_{1}, m_{2}\right)$ we have

$$
\left(v_{m_{1}}, k_{k} v_{m_{2}}\right)=v_{m_{1}} \otimes f^{(k)} v_{m_{2}} \bmod q^{-1 / 4} \mathscr{L} .
$$

This equality easily implies that $\mathscr{L}=\mathscr{L}_{T}$ and then $\mathscr{B}=\mathscr{B}_{T}$. 
(1.3.13) Corollary. For $k_{1}+k_{2} \leqq m_{2}$,

$$
\left\{\begin{array}{ccc}
m_{1} & m_{2} & m_{3} \\
k_{1} & k_{2} & k
\end{array}\right\}_{q}=\left\{\begin{array}{cl}
1 \bmod q^{-1 / 4} A & \text { if } k=k_{1} \\
0 \bmod q^{-1 / 4} A & \text { otherwise } .
\end{array}\right.
$$

For $k_{1}+k_{2}>m_{2}$

$$
\left\{\begin{array}{ccc}
m_{1} & m_{2} & m_{3} \\
k_{1} & k_{2} & k
\end{array}\right\}_{q}=\left\{\begin{array}{cl}
1 \bmod q^{-1 / 4} A & \text { if } k=m_{2}-k_{1}, \\
0 \bmod q^{-1 / 4} A & \text { otherwise. }
\end{array}\right.
$$

(1.3.14) Remark. For a finite dimensional $U_{q}$-module $M$, we have ker $e=$ $\bigoplus_{\lambda} \operatorname{ker} e \cap M_{\lambda}$.

$A$ crystal base of kere of $M$ is a pair $\left(L_{\lambda}, B_{\lambda}\right)$ for each $\lambda$ such that kere $\cap$ $M_{i} \neq\{0\}$. A pair $\left(L_{\lambda_{i}}, B_{\lambda}\right)$ has to satisfy the following conditions.

$L_{\lambda_{2}}$ is a free sub- $A$-module of kere $\cap M_{\lambda_{2}}$ such that

$$
\text { kere } \cap M_{\lambda}=\mathbb{Q}\left(q^{1 / 4}\right) \underset{A}{\bigotimes} L_{\lambda} \text {. }
$$

$B$ is a base of the $\mathbb{Q}$-vector space $L_{\lambda} / q^{1 / 4} L_{\lambda}$.

Obviously, a crystal base of ker $e$ is uniquely extended to a crystal base of $M$. By (1.3.9), any crystal base of $M$ is the extension of a crystal base on kere.

(1.4). Bases in Tensor Products of $s l_{2}$-Modules. Consider the Lie algebra $\mathrm{g}=s l_{2}$ with the generators $e, f, h$ such that

$$
[e, f]=h, \quad[h, e]=2 e, \quad[h, f]=-2 f .
$$

Denote by $\Omega$ the Casimir element

$$
\frac{1}{2} h \otimes h+e \otimes f+f \otimes e \in \mathfrak{g} \otimes \mathfrak{g} .
$$

Let $M_{1}, \ldots, M_{n}$ be $g$ modules. For $i<j$ let $\Omega_{l j}$ be the linear operator on $M_{1} \otimes$ $\cdots \otimes M_{n}$ acting as $\Omega$ on $M_{l} \otimes M_{j}$ and as the identity operator on the other factors.

For a $\mathfrak{g}$-module $M$ and $\lambda \in \mathbb{C}$, let $M_{\lambda}=\{u \in M \mid h u=\lambda u\}$ be the space of weight $\lambda$ and Sing $M_{\lambda}=$ kere $\cap M_{\lambda}$ the space of singular elements of weight $\lambda$.

For a nonnegative integer $m$, let $L(m)$ be the $(m+1)$ dimensional irreducible $\mathrm{g}$-module. The module is generated by its singular element $v_{m}$ such that $e v_{m}=0$ and $h v_{m}=m v_{m}$. The elements $v_{m}, f v_{m}, \ldots, f^{m} v_{m}$ form a basis of $L(m)$.

The Shapovalov form on $L(m)$ is the bilinear form $B^{m}$ such that

$$
B^{m}\left(f^{k} v_{m}, f^{k} v_{m}\right)=k ! m ! /(m-k) !, \quad B^{m}\left(f^{k} v_{m}, f^{l} v_{m}\right)=0 \quad \text { for } k \neq l .
$$

The tensor product of irreducible $g$ modules is the direct sum of irreducible modules: $L\left(m_{1}\right) \otimes L\left(m_{n}\right)=L\left(\left|m_{1}-m_{2}\right|\right) \otimes L\left(\left|m_{1}-m_{2}\right|+2\right) \otimes \cdots \otimes L\left(m_{1}+m_{2}\right)$, and a singular vector of $L\left(m_{1}+m_{2}-2 k\right)$ can be chosen of the form

$$
\left\{v_{m_{1}},{ }_{k} v_{m_{2}}\right\}=\sum_{p=0}^{k}(-1)^{p} \frac{\prod_{j=0}^{k-1}\left(m_{1}+m_{2}-2 k+j+2\right)}{\prod_{j=0}^{p-1}\left(m_{1}-j\right) \prod_{j=0}^{k-p-1}\left(m_{2}-j\right)} f^{p} v_{m_{1}} \otimes f^{k-p} v_{m_{2}} .
$$


This decomposition gives a basis $\left\{f^{l}\left\{v_{m_{1}},{ }_{k} v_{m_{2}}\right\}\right\}$ in the tensor product. We have

$$
\begin{aligned}
\Omega f^{l}\left\{w_{m_{1}}, k v_{m_{2}}\right\} & =\mu\left(m_{1}, m_{2} ; k\right) \cdot f^{l}\left\{w_{m_{1}}, k v_{m_{2}}\right\}, \\
\mu\left(m_{1}, m_{2} ; k\right) & =\frac{1}{2} m_{1} m_{2}-k\left(m_{1}+m_{2}\right)+k(k-1) .
\end{aligned}
$$

For nonnegative integers $m_{1}, \ldots, m_{n}$, consider the tensor product $L=L\left(m_{1}\right) \otimes$ $\cdots \otimes L\left(m_{n}\right)$. For any $n$-tree $T$ the tensor product has a distinguished basis $\mathscr{B}_{T}=$ $\{v(c, l, T)\}$. The elements of $\mathscr{B}_{T}$ are numerated by the pairs $(c, l)$, where $c$ is an admissible coloring of $T$ and $l=0, \ldots, m(c)$, see (1.3). The vector $v(c, l, T)$ is defined inductively on $n$ as in (1.3) but we use the formula

$$
v(c, l, T)=f^{l}\left\{v^{l e}, c(w) v^{r}\right\}
$$

instead of formula (1.2.9).

For any $n$-tree $T$ and a permutation $\sigma \in S_{n}$ define a basis $\mathscr{B}_{T, \sigma}=\{v(c, l, T, \sigma)\}$ in $L\left(m_{1}\right) \otimes \cdots \otimes L\left(m_{n}\right)$. Namely, let $P_{\sigma}: L\left(m_{1}\right) \otimes \cdots \otimes L\left(m_{n}\right) \rightarrow L\left(m_{\sigma(1)}\right) \otimes \cdots \otimes$ $L\left(m_{\sigma(n)}\right)$ be the permutation of factors. Let $\mathscr{B}_{T}=\{v(c, l, T)\}$ be the basis in $L\left(m_{\sigma(1)}\right) \otimes \cdots \otimes L\left(m_{\sigma(n)}\right)$ corresponding to the tree $T$ with tops marked by $m_{\sigma(1)}, \ldots$, $m_{\sigma(n)}$. Set $v(c, l, T, \sigma)=P_{\sigma}^{-1} v(c, l, T)$ for all $c, l$.

For any $T, \sigma$ and an internal vertex $w$ of $T$, define an operator $\Omega_{w, T, \sigma}$ acting on $L\left(m_{1}\right) \otimes \cdots \otimes L\left(m_{n}\right)$. Namely, for an internal vertex $w$, let $J$ be the set of indices of the tops of $T$ which belong to the right branch or the left branch of $w$. Set

$$
\Omega_{w, T, \sigma}=\sum_{\substack{i<j \\ l, J \in \sigma^{-1}(J)}} \Omega_{l j}
$$

For every $T, \sigma, w$ the basis $\mathscr{B}_{T, \sigma}$ is an eigenbasis for the operator $\Omega_{w, T, \sigma}$ :

$$
\Omega_{w, T, \sigma} v(c, l, T, \sigma)=\mu(c, T, \sigma, w) \cdot v(c, l, T, \sigma)
$$

for all $c, l$ and suitable numbers $\mu(c, T, \sigma, w)$, cf. (1.4.2).

(1.5). The Selberg Integral and Normalizing Constants. For any colored tree we define a function called the normalizing constant.

The Selberg integral is the integral

$$
I_{k}(a, b ; \kappa)=k ! \int \prod_{\Delta=1}^{k} t_{j}^{-a / \kappa}\left(1-t_{j}\right)^{-b / \kappa} \prod_{1 \leqq l<j \leqq k}\left(t_{j}-t_{i}\right)^{2 / \kappa} d t_{1} \wedge \cdots \wedge d t_{k},
$$

where $\Delta=\left\{t \in \mathbb{R}^{k} \mid 0<t_{1}<\cdots<t_{k}<1\right\}$. The integral can be computed explicitly $[\mathrm{M}]$ :

$$
I_{k}(a, b ; \kappa)=\prod_{j=0}^{k-1} \frac{\Gamma\left(\frac{-a+j}{\kappa}+1\right) \Gamma\left(\frac{-b+j}{\kappa}+1\right) \Gamma\left(\frac{i+1}{\kappa}+1\right)}{\Gamma\left(\frac{-a-b+2 k-j-2}{\kappa}+2\right) \Gamma\left(\frac{1}{\kappa}+1\right)} .
$$

Set

$$
q(\kappa)=\exp (2 \pi i / \kappa)
$$


Define $J_{k}(a, b ; \kappa)$ by

$$
\begin{aligned}
J_{k}(a, b ; \kappa)= & (2 \pi \kappa)^{-k / 2} q(\kappa)^{\frac{-k b}{2}+\frac{3}{4} k(k-1)} I_{k}(a, b, \kappa) \\
& \cdot \prod_{j=0}^{k-1}(1-\kappa /(a+b-2 k+j+2)) \cdot(b-j)_{q(\kappa)},
\end{aligned}
$$

where $q$-numbers ()$_{q}$ are defined in (1.1.3).

Let $T$ be an $n$-tree with tops marked by some nonnegative integers $m_{1}, \ldots, m_{n}$. Let $c$ be an admissible coloring of $c$.

For an internal vertex $w \in \operatorname{In}_{T}$ define its normalizing constant $C(w, c, T, \kappa)$ by

$$
C(w, c, T, \kappa)=J_{c(w)}\left(m^{l e}(w), m^{r}(w) ; \kappa\right),
$$

where $c(w)$ is the color of $w$ with respect to $c$, and $m^{l e}(w)$ (resp. $m^{r}(w)$ ) is the weight of the left (resp. right) branch of $w$, see (1.2).

Define the normalizing constant, $C(c, T, \kappa)$, of a coloring $c$ by the rule

$$
C(c, T, \kappa)=\left(\sum_{w \in \operatorname{In}_{T}} c(w)\right) ! \prod_{w \in \operatorname{In}_{T}} C(w, c, T, \kappa) / c(w) !
$$

\section{Asymptotic Solutions to the Knizhnik-Zamolodchikov Equation}

(2.1). Knizhnik-Zamolodchikov Equation. Let $M_{1}, \ldots, M_{n}$ be $\mathfrak{g}=s l_{2}$ modules, $M=M_{1} \otimes \cdots \otimes M_{n}$. The Knizhnik-Zamolodchikov equation (KZ) on an $M$-valued function $\psi\left(z_{1}, \ldots, z_{n}\right)$ is the system of equations

$$
\kappa \frac{\partial \psi}{\partial z_{l}}=H_{i} \psi, \quad i=1, \ldots, n
$$

where $\kappa$ is a parameter of the equation and

$$
H_{i}=\sum_{j \neq i} \frac{\Omega_{i j}}{z_{i}-z_{j}} .
$$

The $\mathrm{KZ}$ equation defines a connection on the trivial bundle $M \times \mathbb{C}^{n} \rightarrow \mathbb{C}^{n}$ with singularities at diagonal hyperplanes. This connection is flat. Parallel translation with respect to this connection commutes with the $g$ action on $M$.

For a solution $\psi$ to the $\mathrm{KZ}$ equation we have

$$
\left(\frac{\partial}{\partial z_{1}}+\cdots+\frac{\partial}{\partial z_{n}}\right) \psi=0
$$

hence $\psi$ depends only on differences $z_{l}-z_{j}$ of variables.

For nonnegative integers $m_{1}, \ldots, m_{n}$, let $L=L\left(m_{1}\right) \otimes \cdots \otimes L\left(m_{n}\right)$. For any $\lambda$ the $\mathrm{KZ}$ equation with values in $L$ preserves $\operatorname{Sing} L_{\lambda}$. The $\mathrm{KZ}$ equation with values in $\bigoplus_{\lambda} \operatorname{Sing} L_{\lambda}$ determines the $\mathrm{KZ}$ equation on $L$. 
Denote by $B$ the bilinear form $B^{m_{1}} \otimes \cdots \otimes B^{m_{n}}$ on $L$, where $B^{m}$ is the Shapovalov form on $L(m)$, see (1.4). The operators $H_{1}, \ldots, H_{n}$ are $B$-symmetric:

$$
B\left(H_{i} x, y\right)=B\left(x, H_{i} y\right)
$$

for all $i$ and all $x, y \in L[\mathrm{RV}]$.

(2.2). Asymptotic Zones. For $\sigma \in S_{n}$ let

$$
D_{\sigma}=\left\{z \in \mathbb{R}^{n} \mid z_{\sigma(1)}<\cdots<z_{\sigma(n)}\right\} .
$$

For $\sigma \in S_{n}$ and an $n$-tree $T$, we define a diffeomorphism

$$
u_{T, \sigma}: D_{\sigma} \rightarrow \mathbb{R} \times\left(\mathbb{R}_{>0}\right)^{n-1}
$$

called an asymptotic zone.

The first coordinate of $u_{T, \sigma}$ is $z_{1}+\cdots+z_{n}$, the other coordinates are numerated by internal vertices of $T$. Denote by $u_{\omega, T, \sigma}$ the coordinate corresponding to a vertex $\omega$.

Let $\sigma=i d$. The coordinate $u_{\omega}, T$, id is defined inductively. For an internal vertex $\omega$, let $W$ be the set of internal vertices of $T$ belonging to the shortest path in $T$ from $\omega$ to the root. $W$ can be empty. Assume that for any $y \in W$ the coordinate $u_{\omega, T}$, id is already defined. Let $J^{l e}$ (resp. $J^{r}$ ) be the set of the tops of $T$ belonging to the left (resp. right) branch of $w$. Set $j^{l e}=\max \left\{j \in J^{l e}\right\}, j^{r}=\min \left(j \in J^{r}\right)$,

$$
u_{w, T, \text { id }}=\left(z_{j^{r}}-z_{j l e}\right) / \prod_{y \in W} u_{y, T, \text { id }} .
$$

For $\sigma \in S_{n}$, let

$$
\tau_{\sigma}: D_{\sigma} \rightarrow D_{\mathrm{id}}, \quad\left(z_{1}, \ldots, z_{n}\right) \mapsto\left(z_{\sigma(1)}, \ldots, z_{\sigma(n)}\right)
$$

Set

$$
u_{w, T, \sigma}=u_{w, T, \text { id }} \circ \tau_{\sigma} .
$$

Let

$$
\mathscr{U}_{n}=\left\{z_{1}, \ldots, z_{n} \in \mathbb{C}^{n} \mid z_{i} \neq z_{j} \text { for all } i, j\right\}
$$

(2.2.2). Proposition. For every $\sigma, T$ the functions $z_{1}+\cdots+z_{n},\left\{u_{w}, T, \sigma\right\}_{w \in \operatorname{In}_{T}}$ define a diffeomorphism $u_{T, \sigma}$ of $D_{\sigma}$ onto $\mathbb{R} \times\left(\mathbb{R}_{>0}\right)^{n-1}$. The map $u_{T, \sigma}$ is extended to a biholomorphic map of $\mathscr{U}_{n}$ to $\mathbb{C} \times\left(\mathbb{C}^{*}\right)^{n-1}$. Furthermore, $u_{T, \sigma}^{-1}$ is extended to a polynominal map of $\mathbb{C}^{n}$ to $\mathbb{C}^{n}$.

Now we will construct curves connecting the domains $D_{\sigma}$ and lying in $\mathscr{U}_{n}$.

For $j \in\{1, \ldots, n-1\}$ let $\tau$ be the transposition of $j$ and $j+1$. Let $T$ be a tree adjacent to $\tau$, see (1.2), and $w$ the internal vertex of $T$ such that the $j^{\text {th }}$ top of $T$ (resp. the $(j+1)^{\text {th }}$ ) forms the left (resp. right) branch of $w$.

For any permutation $\sigma$, the biholomorphisms $u_{T, \sigma}$ and $u_{T, \sigma \tau}$ have the property:

$$
\begin{aligned}
& u_{w, T, \sigma}=-u_{w, T, \sigma \tau} \\
& u_{y, T, \sigma}=u_{y, T, \sigma \tau} \text { for every internal vertex } y \text { different from } w .
\end{aligned}
$$


Define a curve $\gamma_{T, \sigma, \tau}:[0,1] \rightarrow \mathscr{U}_{n}$ by the rule:

$$
\begin{aligned}
& u_{w, T, \sigma}=\exp (\mathrm{i} t) \text { for } t \in[0,1], \\
& u_{y, T, \sigma}=1 \text { for all } t \in[0,1] \text { and all } y \in \operatorname{In}_{T} \text { such that } y \neq w .
\end{aligned}
$$

We have $\gamma(0) \in D_{\sigma}$ and $\gamma(1) \in D_{\tau \sigma}$. The curve $\gamma_{T, \sigma, \tau}$ forms a half circle connecting $D_{\sigma}$ and $D_{\sigma \tau}$. The complementary half circle is formed by the curve $\gamma_{T, \sigma \tau, \tau}$.

For every $T, \sigma$ the biholomorphism $u_{T, \sigma}$ resolves singularities of the union of hyperplanes in the following sense. Let

$$
f: \mathbb{C}^{n} \rightarrow \mathbb{C}, \quad\left(z_{1}, \ldots, z_{n}\right) \mapsto \prod_{i<j}\left(z_{i}-z_{j}\right) .
$$

Then for every $\sigma, T$ we have

$$
f \circ u_{T, \sigma}^{-1}=\prod_{w \in \operatorname{In}_{T}}\left(u_{w, T, \sigma}\right)^{a_{w}} \cdot(1+\mathcal{O}(u))
$$

for suitable positive integers $\left\{a_{w}\right\}$.

Consider the $\mathrm{KZ}$ equation with values in $V=V_{1} \otimes \cdots \otimes V_{n}$.

(2.2.3). Proposition. For any $\sigma, T$, the $K Z$ equation with respect to variables $z_{1}+$ $\cdots+z_{n}$ and $\left\{u_{w}, T, \sigma\right\}$ has the form

$$
\begin{gathered}
\left(\frac{\partial}{\partial z_{1}}+\cdots+\frac{\partial}{\partial z_{1}}\right) \psi=0, \\
\frac{\partial \psi}{\partial u_{w, T, \sigma}}=\frac{1}{\kappa}\left(\frac{\Omega_{w, T, \sigma}}{u_{w, T, \sigma}}+\operatorname{Reg}\right) \psi,
\end{gathered}
$$

where Reg is an $\operatorname{End}(V)$-valued function holomorphic at $u_{T, \sigma}=0$, and $\Omega_{w, T, \sigma}$ is the constant operator defined in (1.4.4).

Example. For the tree in (1.2.7) and the identity permutation, the coordinates are $z_{1}+z_{2}+z_{3}, u_{1}=z_{3}-z_{2}, u_{2}=\left(z_{2}-z_{1}\right) /\left(z_{3}-z_{2}\right)$, and the KZ equation has the form

$$
\frac{\partial \psi}{\partial u_{1}}=\frac{1}{\kappa}\left(\frac{\Omega_{12}+\Omega_{13}+\Omega_{23}}{u_{1}}+\operatorname{Reg}\right), \quad \frac{\partial \psi}{\partial u_{2}}=\frac{1}{\kappa}\left(\frac{\Omega_{12}}{u_{2}}+\operatorname{Reg}\right) .
$$

(2.3). Asymptotic Solutions. For $m_{1}, \ldots, m_{n} \in \mathbb{N}$ and a nonnegative integer $\lambda$, consider the tensor product of irreducible $s l_{2}$ modules $L=L\left(m_{1}\right) \otimes \cdots \otimes L\left(m_{n}\right)$ and the $\mathrm{KZ}$ equation with values in $\operatorname{Sing} L_{\lambda}$. Throughout the remainder of the paper we assume that the parameter $\kappa$ of the $\mathrm{KZ}$ equation is not a rational number.

For any $n$-tree $T$ and a permutation $w \in S_{n}$ we will construct a fundamental system of solutions, $\psi_{T, \sigma}$, to the $\mathrm{KZ}$ equation.

Namely, consider the basis $\mathscr{B}_{T, \sigma}$ of $L$. Then.

$$
\mathscr{B}_{T, \sigma, \lambda}:=\mathscr{B}_{T, \sigma} \cap \operatorname{Sing} L_{\lambda}
$$

is a basis of $\operatorname{Sing} L_{\lambda}$. Vectors of $B_{T, \sigma, i}$ have the form $v(c, 0, T, \sigma)$, see (2.1), where $c$ is an admissible coloring of the tree $T$ with tops marked by $m_{\sigma(1)}, \ldots, m_{\sigma(n)}$ and the coloring $c$ has weight $\lambda$, see (1.2.8). Denote the set of such colorings by 
$\operatorname{Adm}(T, \sigma, \lambda)$. A vector $v(c, 0, T, \sigma)$ is an eigenvector of the operators $\left\{\Omega_{w, T, \sigma}\right\}_{w \in \operatorname{In}_{T}}$ with eigenvalues denoted by $\{\mu(c, T, \sigma, w)\}$, resp., see (1.4.5).

For every such a vector there exists a unique solution, $\psi_{c, T, \sigma}$, to the $\mathrm{KZ}$ equation restricted to $D_{\sigma}$ such that

$$
\psi_{c, T, \sigma}=C(c, T, \kappa)\left(\prod_{w \in \operatorname{In} T}\left(u_{w, T, \sigma}\right)^{\mu(c, T, \sigma, w) / \kappa}\right) \cdot\left(v(c, 0, T, \sigma)+\mathcal{O}\left(u_{T, \sigma}, \kappa\right)\right),
$$

where $C(c, T, \kappa)$ is the normalizing constant of the colored tree $T$ with tops marked by $m_{\sigma(1)}, \ldots, m_{\sigma(n)}, \mathcal{O}\left(u_{T, \sigma}, \kappa\right)$ is a $\operatorname{Sing} L_{\lambda}$-valued function which is regular function of $u_{T, \sigma}$ at $u_{T, \sigma}=0$ and which tends to zero as all coordinates $u_{T, \sigma}$ tend to zero, univalued branches of the functions $\left\{u^{\mu}\right\}$ are chosen by the rule: $\arg \left(u_{w}, T, \sigma\right)=0$ for all $w \in \operatorname{In}_{T}$.

The collection of these solutions form a fundamental system of solutions. It will be called the asymptotic solution corresponding to the asymptotic zone $u_{T, \sigma}$, and will be denoted by $\psi_{T, \sigma}$.

The asymptotic solution can be analytically continued to a system of multivalued solutions over $\mathscr{U}_{n}$.

(2.4). Transition Functions Between Asymptotic Solutions. The first main result of this paper describes transition functions between asymptotic solutions.

To compare two asymptotic solutions $\psi_{T, \sigma}$ and $\psi_{T^{\prime}, \sigma^{\prime}}$ we have to distinguish a curve from $D_{\sigma}$ to $D_{\sigma}^{\prime}$, lying in $\mathscr{U}_{n}$, then analytically continue $\psi_{T, \sigma}$ along the curve and express the analytic continuation in terms of $\psi_{T^{\prime}, \sigma^{\prime}}$.

Let $T$ and $T^{\prime}$ be $n$-trees. Let $w_{1}$ and $w_{2}$ be internal vertices of $T$ and $w_{1}^{\prime}$ and $w_{2}^{\prime}$ internal vertices of $T^{\prime}$. Assume that $T$ and $T^{\prime}$ are adjacent at $w_{1}, w_{2}, w_{1}^{\prime}, w_{2}^{\prime}$, see (1.2).

For any $\sigma \in S_{n}$, the asymptotic solutions $\psi_{T, \sigma}$ and $\psi_{T^{\prime}, \sigma}$ are defined over the same $D_{\sigma}$.

(2.4.1). Theorem. For any $c \in \operatorname{Adm}(T, \sigma, \lambda)$ we have

$$
\psi_{c, T, \sigma}=\sum_{\substack{c^{\prime} \equiv c \\
c^{\prime} \in \operatorname{Adm}\left(T^{\prime}, \sigma, i\right)}}\left\{\begin{array}{ccc}
m^{l e}\left(w_{1}\right) & m^{r}\left(w_{1}\right) & m^{r}\left(w_{2}\right) \\
c\left(w_{1}\right) & c\left(w_{2}\right) & c^{\prime}\left(w_{1}^{\prime}\right)
\end{array}\right\}_{q(\kappa)} \psi_{c^{\prime}, T^{\prime}, \sigma}
$$

Here \{\}$_{q(\kappa)}$ are the $6 j$-symbols for $q(\kappa)=\exp (2 \pi i / \kappa)$. For the definition of the arguments of the $6 j$-symbols, see (1.2).

The theorem is proved in Sect. 4.

For $j \in\{1, \ldots, n-1\}$, let $\tau$ be the transposition of $j$ and $j+1$. Let $T$ be an $n$-tree adjacent to $\tau$, see (1.2), and $w$ the internal vertex of $T$ such that the $j^{\text {th }}$ tops of $T$ (resp. the $(j+1)$ ) forms the left (resp. right) branch of $w$.

For any $\sigma \in S_{n}$, the curve $\gamma_{T, \sigma, \tau}$, defined in (2.2), connects $D_{\sigma}$ and $D_{\sigma \tau}$. Continue $\psi_{T, \sigma}$ along the curve and express the continuation in terms of $\psi_{T, \sigma \tau}$.

(2.4.2). Proposition. For any $c \in \operatorname{Adm}(T, \sigma, \lambda)$ we have

$$
\psi_{c, T, \sigma}=R\left(m_{\sigma(j)}, m_{\sigma(j+1)} ; c(w)\right)_{q(\kappa)} \psi_{c, T, \sigma \tau},
$$

where $R$ is defined in (1.2.3).

The proposition easily follows from the definition of the normalizing constant. 
Statements (2.4.1) and (2.4.2) allow us to compare two arbitrary asymptotic solutions.

By (2.4.1) and (2.4.2), the transition functions between asymptotic solutions to the $\mathrm{KZ}$ equation are exactly the same as the transition functions between the bases in tensor products of irreducible modules over the quantum group $U_{q=q(\kappa)}$, where $U_{q=q(\kappa)}$ is the $\mathbb{C}$-algebra obtained from $U_{q}$ by specializing the indeterminate $q^{1 / 4}$ to $q(\kappa / 4)$. Namely, for natural $m_{1}, \ldots, m_{n}$, let $L\left(m_{1}\right), \ldots, L\left(m_{n}\right)$ be the irreducible $s l_{2}$-modules with highest weights $m_{1}, \ldots, m_{n}$, resp. For a nonrational $\kappa \in \mathbb{C}$ and an integer $\lambda$, consider the $\mathrm{KZ}$ equation with the parameter $\kappa$ and the values in Sing $L_{i}$. For an $n$-tree $T$ and a permutation $\sigma \in S_{n}$, consider the asymptotic solution $\psi_{T, \sigma}(\kappa)=\left\{\psi_{c, T, \sigma}\right\}$.

Let $L\left(m_{1}, q=q(\kappa)\right), \ldots, L\left(m_{n}, q=q(\kappa)\right)$ be irreducible $U_{q=q(n)}$ modules with highest weight $m_{1}, \ldots, m_{n}$, resp. For a permutation $\sigma \in S_{n}$, consider the tensor product

$$
L^{\sigma}=L\left(m_{\sigma(1)}, q=q(\kappa)\right) \otimes \cdots \otimes L\left(m_{\sigma(n)}, q=q(\kappa)\right),
$$

and the subspace

$$
\operatorname{Sing} L^{\sigma}(q=q(\kappa))=\left\{v \in L^{\sigma} \mid q^{h} v=q(\kappa)^{\lambda} v, e v=0\right\} .
$$

Let $\mathscr{B}_{T, \sigma}(q=q(\kappa))=\{v(c, 0, T)\}$ be the basis in $\operatorname{Sing} L^{\sigma}(q=q(\kappa))_{\lambda}$ corresponding to an $n$-tree $T$, see (1.2).

Define a map

$$
\pi_{T, \sigma}(\kappa): \mathscr{B}_{T, \sigma}(q=q(\kappa)) \rightarrow \psi_{T, \sigma}(\kappa), \quad v(c, 0, T) \mapsto \psi_{c, T, \sigma} .
$$

(2.4.6). Corollary of (1.2.12) and (2.4.1). For every $\sigma \in S_{n}$ the maps $\left\{\pi_{T, \sigma}(\kappa)\right\}$ induce a well defined isomorphism $\pi_{\sigma}(\kappa)$ of $\operatorname{Sing} L^{\sigma}(q=q(\kappa))_{i}$ and the space of solutions to the $K Z$ equation over $D_{\sigma}$ with parameters $\kappa$ and values in $\operatorname{Sing} L_{\lambda}$.

(2.4.7). Corollary of (1.2.13) and (2.4.2). Under the isomorphisms described in (2.4.6), the R-matrix action on $\left\{\operatorname{Sing} L^{\sigma}(q=q(\kappa))_{\lambda}\right\}_{\sigma \in S_{n}}$ given by (1.2.13) is isomorphic to the monodromy of the $K Z$ equation with the parameter $\kappa$ and with values in $\operatorname{Sing} L_{i}$.

Kohno [Ko] and Drinfeld [D] proved existence of an isomorphism between the $R$-matrix action and the monodromy representation of the $\mathrm{KZ}$ equation for generic $\kappa$. The case $\kappa=1 / l$ for a natural $l$ is described in [V, Sects. 13-14]. In Sect. 3 we will construct isomorphisms (2.4.6) and (2.4.7) geometrically in terms of integral representations for solutions to the $\mathrm{KZ}$ equation, see $[\mathrm{SV}, \mathrm{V}]$

(2.5). Quasiclassical Asymptotics. Let $D$ be a ball, $\pi: \mathbb{C}^{N} \otimes D \rightarrow D$ projection. Let

$$
\nabla_{\kappa}=\kappa d-\omega
$$

be a holomorphic connection in $\pi$ depending on the parameter $\kappa$. Here

$$
\omega=H_{1} d z_{1}+\cdots+H_{n} d z_{n},
$$

where $\left\{H_{i}\right\}$ are matrix valued functions. Assume that for every $\kappa$ the connection is integrable, $\kappa d \omega+\omega \wedge \omega=0$, or

for all $i$ and $j$.

$$
\frac{\partial H_{i}}{\partial z_{j}}=\frac{\partial H_{j}}{\partial z_{l}}, \quad\left[H_{i}, H_{j}\right]=0
$$


An asymptotically flat section is a section of the form

$$
F=\exp (S / \kappa)\left(f_{0}+\kappa f_{1}+\cdots\right) \text { for } \kappa \rightarrow 0 .
$$

Here $S\left(z_{1}, \ldots, z_{n}\right)$ is a function, $\left\{f_{J}\left(z_{1}, \ldots, z_{n}\right)\right\}$ are sections of $\pi$, and $F$ must be a formal solution of the equation $\nabla_{k} F=0$.

We call $\exp (S / \kappa) f_{0}$ an asymptotically flat section of the first order if there exists a power series (2.5.2) which provides an asymptotic solution to the equation $\nabla_{\kappa} F=0$ modulo terms of order $\kappa^{2}$.

Assume that the linear operators $\left\{H_{J}\right\}$ are simultaneously diagonalizable for each $z \in D$ : there exists a basis $\left\{v_{l}(z)\right\}$ in $\mathbb{C}^{N}$ such that

$$
H_{J}(z) v_{l}(z)=\lambda_{j l}(z) \cdot v_{l}(z)
$$

for all $j, l$. Assume that the spectrum of $\left\{H_{j}\right\}$ separates elements of the basis: for every $z$ and every $l, m$, there exists $j$ such that $\lambda_{j l}(z) \neq \lambda_{j m}(z)$.

Let $F=\exp (S / \kappa) f_{0}$ be an asymptotically flat section of the first order, then $f_{0}$ is an eigenvector of the operators $\left\{H_{i}\right\}$,

$$
H_{J}(z) f_{0}(z)=\lambda_{\jmath}(z) f_{0}(z), \quad j=1, \ldots, n,
$$

moreover, $\lambda_{J}=\frac{\partial S}{\partial z_{j}}$, see $[\mathrm{RV}]$

Let $\exp (S / \kappa) f_{0}$ and $\exp (T / \kappa) g_{0}$ be asymptotically flat sections of the first order corresponding to the same eigenvector of $\left\{H_{i}\right\}$, then

$$
\exp (S / \kappa) f_{0}=\text { const } \cdot \exp (T / \kappa) g_{0}
$$

see $[R V]$.

Assume that there exists a symmetric bilinear form $B: \mathbb{C}^{n} \otimes \mathbb{C}^{n} \rightarrow \mathbb{C}$ and the operators $H_{1}, \ldots, H_{n}$ are symmetric with respect to $B: B\left(H_{i} x, y\right)=B\left(x, H_{l} y\right)$ for all $x, y, i$. Assume that $\exp (S / \kappa)\left(f_{0}+\cdots\right)$ and $\exp (T / \kappa)\left(g_{0}+\cdots\right)$ are two asymptotically flat sections.

(2.5.6). Lemma [RV]. If $S-T \neq$ const, then $B\left(f_{0}, g_{0}\right) \equiv 0$, if $S-T=$ const then $B\left(f_{0}, g_{0}\right) \equiv$ const.

The $\mathrm{KZ}$ equation gives an example of a family of flat connections. The $\mathrm{KZ}$ operators $\left\{H_{i}\right\}$ are symmetric with respect to the Shapovalov form.

For natural $m_{1}, \ldots, m_{n}$, let $L\left(m_{1}\right), \ldots, L\left(m_{n}\right)$ be the irreducible $s l_{2}$ modules with highest weights $m_{1}, \ldots, m_{n}$. For a nonrational number $\kappa$ and an integer $\lambda$, consider the $\mathrm{KZ}$ equation with parameter $\kappa$ and with values in $\operatorname{Sing} L_{\lambda}$. For an $n$-tree $T$ and a permutation $\sigma \in S_{n}$ consider the asymptotic solution $\psi_{T, \sigma}=\left\{\psi_{c, T, \sigma}\right\}$. Consider the coordinates $\left\{u_{w}, T, \sigma\right\}_{w \in \operatorname{In}_{T}}$. These coordinates take positive values on $D_{\sigma}$.

For $\varepsilon>0$, let

$$
D_{\sigma, T, \varepsilon}=\left\{p \in D_{\sigma} \mid u_{w, T, \sigma}(p)<\varepsilon \quad \text { for all } w \in \operatorname{In}_{T}\right\} .
$$

Our second main result is the following theorem.

(2.5.7). Theorem. Assume that $\kappa=i$ and $s \rightarrow+0$. Then there exists $\varepsilon>0$ such that any solution $\psi_{c, T, \sigma} \in \psi_{T, \sigma}$, restricted to $D_{\sigma, T, \varepsilon}$, has an asymptotic expansion

$$
\psi_{c, T, \sigma} \sim \prod_{w \in \ln _{T}}\left(u_{w, T, \sigma}\right)^{-i \mu(c, T, \sigma, w) / s} \exp (-i S / s) \sum_{j=0}^{\infty} f_{j} s^{j}
$$


where $\{\mu\}$ are the numbers described in (2.3.1) and (1.4.5);

$$
S=S_{c, T, \sigma}: D_{\sigma, T, \varepsilon} \rightarrow \mathbb{R}, \quad f_{l}=f_{j, c, T, \sigma}: D_{\sigma, T, \varepsilon} \rightarrow \operatorname{Sing} L_{\lambda}
$$

are suitable real analytic functions, all the functions $\left\{S, f_{j}\right\}$ depend only on $\left\{u_{w}, T, \sigma\right\}_{w \in \operatorname{In}_{T}}$ and can be analytically continued to real analytic functions in a neighborhood of the set $\left\{u_{w, T}, \sigma=0 \mid w \in \operatorname{In}_{T}\right\}$.

The asymptotic expansion can be differentiated an arbitrary number of times.

The asymptotic expansion means that for any $N$,

$$
\left|\psi_{c, T, \sigma} \prod_{w \in \operatorname{In}_{T}}\left(u_{w}\right)^{i \mu(w) / s} \exp (i S / s)-\sum_{j=0}^{\infty} f_{j} s^{j}\right|=\mathcal{O}\left(s^{N+1}\right)
$$

uniformly in $D_{\sigma, T, \varepsilon}$.

The theorem has the following appendix.

(2.5.9). Appendix.

(1) For any $p \in D_{\sigma, T, \varepsilon}$ the first terms $\left\{f_{0, c, T, \sigma}(p)\right\}$ of the above asymptotic expansions form a basis in $\operatorname{Sing} L_{\lambda}$ orthogonal with respect to the Shapovalov form.

(2) For any $c, f_{0, c, T, \sigma}(p)$ tends to

$$
\begin{aligned}
(-1)^{k} k ! & \cdot\left(\prod_{w \in \operatorname{In}_{T}}(c(w) !)^{3} \prod_{j=0}^{c(w)-1} \frac{\left(m^{l e}(w)+m^{r}(w)-2 c(w)+j+2\right)^{3}}{\left(m^{l e}(w)-j\right)\left(m^{r}(w)-j\right)}\right)^{-1 / 2} \\
& \cdot v(c, 0, T, \sigma)
\end{aligned}
$$

as $u_{w, T, \sigma}(p) \rightarrow 0$ for all $w \in \operatorname{In}_{T}$. Here $T$ is an $n$-tree with vertices marked by $m_{\sigma(1)}, \ldots, m_{\sigma(n)}$. The numbers $c(w), m^{l e}(w)$, and $m^{r}(w)$ for an $n$-tree with marked tops are defined in (1.2). The vector $v(c, 0, T, \sigma)$ is defined by (2.3.1), $k=\left(m_{1}+\cdots+m_{n}-\lambda\right) / 2$.

The theorem and the appendix are proved in Sect. 4.

(2.6). Quasiclassical Asymptotics and Crystal Base. For natural $m_{1}, \ldots, m_{n}$, let $L\left(m_{1}, q\right), \ldots, L\left(m_{n}, q\right)$ be irreducible $U_{q}$ modules with highest weights $m_{1}, \ldots, m_{n}$, resp. For any permutation $\sigma \in S_{n}$, let

$$
L^{\sigma}(q)=L\left(m_{\sigma(1)}, q\right) \otimes \cdots \otimes L\left(m_{\sigma(n)}, q\right) .
$$

Statements (2.4.6) and (2.5.7) allow us to give a construction of a crystal base in $L^{\sigma}(q)$ purely in terms of quasiclassical asymptotics of solutions to the $\mathrm{KZ}$ equation.

This "quasiclassical" crystal base coincides with the distinguished crystal base of the tensor product defined in (1.3). This statement can be considered as a quasiclassical characterization of the distinguished crystal base.

We will give a construction for $\sigma=i d$, since for an arbitrary permutation the construction is the same.

To construct a crystal base in $L(q)=L\left(m_{1}, q\right) \otimes \cdots \otimes L\left(m_{n}, q\right)$ it suffices to construct a crystal base in

$$
\operatorname{Sing} L(q)_{\lambda}=\left\{v \in L(q) \mid q^{h} v=q^{\lambda} v, e v=0\right\}
$$

for every $\lambda$. 
Let $\mu: \mathbb{Q}\left(q^{\frac{1}{4}}\right) \rightarrow \frac{1}{4} \mathbb{Z}$ be the valuation map which assigns to a function $g\left(q^{\frac{1}{4}}\right)$ the order of its pole at $q=\infty$.

Using the $\mathrm{KZ}$ equation, we will construct a map

such that

$$
\mu: \operatorname{Sing} L(q)_{i} \rightarrow \frac{1}{4} \mathbb{Z}
$$

$$
\begin{aligned}
\mu(v) & =0 \quad \text { iff } v=0, \\
\mu(v+w) & \leqq \max (\mu(v), \mu(w)), \\
\mu(g v) & =\mu(g)+\mu(v)
\end{aligned}
$$

for all $v, w \in \operatorname{Sing} L(q)_{\lambda}, g \in \mathbb{Q}\left(q^{\frac{1}{4}}\right)$.

Let $v \in \operatorname{Sing} L(q)_{i}$,

$$
v=\sum_{k_{1}, \ldots, k_{n}} a_{K} f^{k_{1}} v_{1} \otimes \cdots \otimes f^{k_{n}} v_{n}
$$

where $v_{J}$ is the generating vector of $L\left(m_{j}, q\right), j=1, \ldots, n$, and the coefficients $\left\{a_{K}\right\}$ are rational functions in $q^{\frac{1}{4}}$.

For $\kappa \in \mathbb{C}$, let $\operatorname{Sing} L(q=q(\kappa))_{\lambda}$ be the space defined by (2.4.4). For any $\kappa$ such that $q=q(\kappa)$ is not a pole of the functions $\left\{a_{K}\right\}$, let $v(q=q(\kappa)) \in \operatorname{Sing} L(q=$ $q(\kappa))$ be the specialization of $v$ at $q=q(\kappa)$.

Consider the $\mathrm{KZ}$ equation with parameter $\kappa$ and values in $\operatorname{Sing} L_{j}$, see (2.3). Let $\operatorname{Sol}(\kappa)$, be the space of its solutions over $D_{\text {id }}$, and let $\pi(\kappa): \operatorname{Sing} L(q=q(\kappa)) \rightarrow$ $\operatorname{Sol}(\kappa) \lambda$ be the isomorphism defined in (2.4.6). Then $y(\kappa)=\pi(\kappa)(v(q=q(\kappa))) \epsilon$ $\operatorname{Sol}(\kappa)_{\lambda}$ is a solution over $D_{\text {id }}$ to the $\mathrm{KZ}$ equation with parameter $\kappa$.

For any $n$-tree $T$, let $\psi_{T, \text { dd }}(\kappa)=\left\{\psi_{c, T \text {, id }}\right\}$ be the asymptotic solution, then

$$
y(\kappa)=\sum_{c} b_{c}\left(q(\kappa)^{\frac{1}{4}}\right) \cdot \psi_{c, T, \sigma},
$$

where $\left\{b_{c}\left(q^{\frac{1}{4}}\right)\right\}$ are some rational functions.

\section{(2.6.2). Proposition.}

1. Let \|\|$: \operatorname{Sing} L_{\lambda} \rightarrow \mathbb{R}_{\geqq 0}$ be any norm on $\operatorname{Sing} L_{\lambda}$. Let $\kappa=i s, s \in \mathbb{R}$, and $s \rightarrow+0$. For any $n$-tree $T$, let $D_{\mathrm{id}, T, \varepsilon} \subset D_{\mathrm{ld}}$ be the domain described in (2.5.7). Then for every $p \in D_{\mathrm{id}, T, \varepsilon}$ there exists a limit of $s \cdot \ln \| y($ is $)(p) \|$. This limit is an element of $\frac{1}{4} \mathbb{Z}$, and does not depend on $p$ and $T$. Denote this limit by $\mu(v)$.

2. The map $\mu$, defined by this rule, has properties (2.8.1).

Proof. The proposition easily follows from (2.5.7), (2.5.9), (2.4.1), and (1.3.12).

Let $A \subset \mathbb{Q}\left(q^{\frac{1}{4}}\right)$ be the subalgebra of functions regular at $q=\infty$. Let

$$
\mathscr{L}_{\lambda}^{0}=\left\{v \in \operatorname{Sing} L(q)_{\lambda} \mid \mu(v) \leqq 0\right\} .
$$

(2.6.3). Corollary. $\mathscr{L}_{\lambda}^{0}$ is an $A$-submodule of $\operatorname{Sing} L(q)_{\lambda}$.

Let $(\mathscr{L}, \mathscr{B})$ be the distinguished crystal base in $L(q)$ defined in (1.3). Let $\left(\mathscr{L}_{\left.\lambda, \mathscr{B}_{\lambda}\right)}\right.$ be the crystal base in $\operatorname{Sing} L(q)_{i}$ induced by $(\mathscr{L}, \mathscr{B})$.

(2.6.4). Proposition. $\mathscr{L}_{\lambda}^{0}=\mathscr{L}_{\lambda}$.

The proposition easily follows from (2.5.7) and (2.5.9). 
For any $n$-tree $T$, let $\psi_{T, \text { id }}=\left\{\psi_{c, T, \text { id }}\right\}$ be the asymptotic solution considered in (2.5.7) and (2.5.9), let

$$
\left\{\prod_{w}\left(u_{w, T, \text { id }}\right)^{-\mu(c, T, \mathrm{id}, w) / s} \exp \left(-i S_{c, T, \mathrm{id}} / s\right) \cdot f_{0, c, T, \mathrm{id}}\right\}
$$

be the collection of its first terms of asymptotics.

Denote by $\operatorname{Asym}_{T}$ the $\mathbb{Q}$-module of $\mathbb{Q}$-linear combinations of the first terms of asymptotics.

(2.6.5). Proposition. The above construction induces the canonical isomorphism of the $\mathbb{Q}$ modules $\mathscr{L}_{i}^{0} / q^{-\frac{1}{4}} \mathscr{L}_{i}^{0}$ and $\mathrm{Asym}_{T}$.

The module $\operatorname{Asym}_{T}$ has a canonical basis generated by the first terms of asymptotics. This basis is uniquely determined by the fact that each element of the basis is the first term of an asymptotic expansion of the form (2.5.8) of a solution to the $\mathrm{KZ}$ equation and by the normalizing condition (2.5.9.2). The canonical basis in $\operatorname{Asym}_{T}$ induces a basis in $\mathscr{L}_{i}^{0} / q^{-\frac{1}{4}} \mathscr{L}_{i}^{0}$. This induced basis coincides with the basis $\mathscr{B}_{i}$.

\section{Integral Representations for Solutions to the $\mathrm{KZ}$ Equation and the Bethe Vectors}

(3.1). Local System. Let $m_{1}, \ldots, m_{n} \in \mathbb{C}, \kappa \in \mathbb{C}^{*}$, let $k$ be a nonnegative integer. Set

$$
\Phi(t, z)=\prod_{1 \leqq l<j \leqq n}\left(z_{l}-z_{l}\right)^{m_{l} m_{j} / 2 \kappa} \prod_{1 \leqq l<j \leqq k}\left(t_{l}-t_{j}\right)^{2 / \kappa} \cdot \prod_{l=1}^{k} \prod_{j=1}^{n}\left(z_{j}-t_{l}\right)^{-m_{J} / \kappa} .
$$

$\Phi$ is a multivalued holomorphic function on

$$
\mathscr{U}_{k+n}=\left\{(t, z) \in \mathbb{C}^{k+n} \mid t_{l} \neq t_{m}, t_{l} \neq z_{m}, z_{l} \neq z_{m}, \text { for all } l, m\right\} .
$$

Univalued branches of $\Phi$ over open subsets of $\mathscr{U}_{k+n}$ generate a complex onedimensional local system over $\mathscr{U}_{k+n}$ denoted by $\mathscr{S}(\kappa)$.

Let $\mathscr{U}_{n}=\left\{z \in \mathbb{C}^{n} \mid z_{l} \neq z_{j}\right.$ for all $\left.l, j\right\}$, and let

$$
p r_{k, n}: \mathscr{U}_{k+n} \rightarrow \mathscr{U}_{n}, \quad(t, z) \mapsto z,
$$

be a projection. Denote its fiber $p r^{-1}(z)$ by $\mathscr{U}_{k, n}(z)$. Denote by $\mathscr{H}_{k, n}(\kappa)$ the complex vector bundle over $\mathscr{U}_{n}$ with fiber $H_{k}\left(\mathscr{U}_{k, n}(z), \mathscr{S}(\kappa)\right)$ over $z \in \mathscr{U}_{n}$. The bundle has a canonical flat connection called the Gauss-Manin connection.

The symmetric group $S_{k}$ acts on $\mathscr{U}_{k+n}$ by permutations of coordinates $t_{1}, \ldots, t_{k}$ preserving fibers of the projection. The function $\Phi$ is symmetric with respect to this action. Therefore, $S_{k}$ naturally acts on the singular chains in $\mathscr{U}_{k, n}(z)$ with coefficients in $\mathscr{S}(\kappa)$. This action induces an action of $S_{k}$ on $H_{k}\left(\mathscr{U}_{k, n}(z), \mathscr{S}(\kappa)\right)$. This action on fibers of $\mathscr{H}_{k, n}(\kappa)$ commutes with the Gauss-Manin connection.

Denote by $H_{k}\left(\mathscr{U}_{k, n}(z), \mathscr{S}(\kappa)\right)_{\text {- }}$ the skew symmetric part of this action:

$$
H_{k}\left(\mathscr{U}_{k, n}(z), \mathscr{S}(\kappa)\right)_{-}=\left\{v \in H_{k} \mid \sigma v=(-1)^{|\sigma|} v \text { for all } \sigma \in S_{k}\right\} .
$$


(3.2). Integral Representations. For $m_{1}, \ldots, m_{n} \in \mathbb{N}$ and a nonnegative integer $\lambda$, consider the tensor product of irreducible $s l_{2}$ modules, $L=L\left(m_{1}\right) \otimes \cdots \otimes L\left(m_{n}\right)$, and the $\mathrm{KZ}$ equation with parameter $\kappa$ and values in $\operatorname{Sing} L_{\lambda}$. We will describe integral representations for solutions to the $\mathrm{KZ}$ equation.

Set $k=\left(m_{1}+\cdots+m_{n}-\lambda\right) / 2$. The number $k$ is a nonnegative integer. A monomial of weight $\lambda$ is an element of $L_{\lambda}$ of the form

$$
f_{K}=f^{k_{1}} v_{1} \otimes \cdots \otimes f^{k_{n}} v_{n}
$$

where $K=\left(k_{1}, \ldots, k_{n}\right), k_{1}+\cdots+k_{n}=k$. For a monomial $f_{K}$ define a differential $k$-form in $t$ and $z$ :

$$
\begin{aligned}
\eta\left(f_{K}\right) & =A_{K}(t, z) d t_{1} \wedge \cdots \wedge d t_{k}, \\
A_{k} & =\sum_{\sigma \in S\left(k ; k_{1}, \ldots, k_{n}\right)} \prod_{i=1}^{k} \frac{1}{\left(t_{1}-z_{\sigma(i)}\right)} .
\end{aligned}
$$

The sum is over the set $S\left(k ; k_{1}, \ldots, k_{n}\right)$ of maps $\sigma$ from $\{1, \ldots, k\}$ to $\{1, \ldots, n\}$ such that for all $m$ the cardinality of $\sigma^{-1}(m)$ is $k_{m}$.

Consider the $L_{\lambda}$-valued form

$$
N=\sum_{f_{K} \in L_{\curlywedge}} \Phi(t, z) \cdot \eta\left(f_{K}\right) \otimes f_{K},
$$

where $\Phi$ is the function defined by (3.1.1). $N$ is a multi-valued holomorphic $k$-form on $\mathscr{U}_{k+n}$.

In $[\mathrm{SV}]$ it is proved that:

For every $j$, the form

$$
\left(\kappa \frac{\partial}{\partial z_{j}}-\sum_{l \neq j} \frac{\Omega_{j, l}}{z_{J}-z_{l}}\right) N
$$

is a sum of the differential of a suitable $(k-1)$-form and a form which has zero restriction to fibers of the projection $p r_{k, n}$.

The form $e N=\sum \Phi \cdot \eta\left(f_{K}\right) \otimes e f_{K}$ is a sum of the differential of a suitable $(k-1)$-form and a form which has zero restriction to fibers of $p r_{k, n}$. (3.2.5)

The forms mentioned in (3.2.4) and (3.2.5) have the shape $\sum \Phi w(M) \otimes M$, where the sum is over monomials in $L$, and $\{w(M)\}$ are suitable rational forms, regular on $\mathscr{U}_{k+n}$.

Assume that $\gamma(z) \in H_{k}\left(\mathscr{U}_{k, n}(z), \mathscr{S}(\kappa)\right)$. Assume that the map $z \mapsto \gamma(z)$ forms a flat section of the bundle $\mathscr{H}_{k, n}(\kappa)$ when $z$ runs through an open subset of $\mathscr{U}_{n}$. Then the function

$$
\Psi(z)=\int_{\gamma(z)} N
$$

takes values in $\operatorname{Sing} L_{\lambda}$ and satisfies the $\mathrm{KZ}$ equation with parameter $\kappa[\mathrm{SV}, \mathrm{V}]$.

(3.2.8). Remark. The group $S_{k}$ naturally acts on the space of differential forms on $\mathscr{U}_{k+n}$ by permutations of $t_{1}, \ldots, t_{k}$. The differential form $N$ is skew symmetric 
with respect to this action. Let $v: H_{k}\left(\mathscr{U}_{k, n}(z), \mathscr{S}(\kappa)\right) \rightarrow H_{k}\left(\mathscr{U}_{k, n}(z), \mathscr{S}(\kappa)\right)_{-}$be the canonical projection, then for any flat section $z \mapsto \gamma(z)$ of $\mathscr{H}_{k, n}(\kappa)$ we have

$$
\int_{\gamma(z)} N=\int_{v(\gamma(z))} N
$$

(3.3). Quasiclassical Aysmptotic Solutions to the $K Z$ Equation. Let $\kappa \rightarrow 0$. We use the form $N$ defined in (3.2.3) to construct quasiclassical asymptotic solutions to the $\mathrm{KZ}$ equation.

The function $\Phi$ can be written in the form

$$
\Phi(t, z)=\exp (S(t, z) / \kappa)
$$

where

$$
\begin{aligned}
S(t, z)= & \sum_{1 \leqq l<j \leqq n} \frac{m_{l} m_{j}}{2} \ln \left(z_{j}-z_{l}\right)+\sum_{1 \leqq l<j \leqq n} 2 \ln \left(t_{l}-t_{j}\right) \\
& -\sum_{l=1}^{k} \sum_{j=1}^{n} m_{j} \ln \left(z_{j}-t_{l}\right) .
\end{aligned}
$$

Set

$$
\operatorname{Hess}_{t}(-S)=\operatorname{det}\left(-\frac{\partial^{2} S}{\partial t_{l} \partial t_{J}}\right) .
$$

For a fixed $z \in \mathscr{U}_{n}$, consider the equation of critical points of $S$ in $\mathscr{U}_{k, n}(z)$ :

$$
\frac{\partial S}{\partial t_{j}}=0, \quad j=1, \ldots, k
$$

Let $t=t(z)$ be a nondegenerate solution of (3.3.3) holomorphically depending on $z$ in a neighborhood of a point $z^{0} \in \mathscr{U}_{n}$.

Let $B(z) \subset \mathscr{U}_{k, n}(z)$ be a small ball with center at $(t(z), z)$. Set

$$
B_{-}(z)=\left\{(t, z) \in \mathscr{U}_{k, n}(z) \mid \operatorname{Im}(S(t, z))<\operatorname{Im}(S(t(z), z))\right\} .
$$

It is known that $H_{k}\left(B(z), B_{-}(z), \mathscr{S}(\kappa)\right)$ is one-dimensional, see Sect. 11 in [AGV]. The Gauss-Manin connection identifies these groups for neighboring $z$ 's.

A generator of the homology group (for all $\kappa$ simultaneously) can be chosen as follows. Fix a branch of arguments of all functions $t_{j}-t_{l}, t_{j}-z_{l}, z_{l}-z_{m}$ for all $l, m$ in a neighborhood of the point $\left(t\left(z^{0}\right), z\right)$. This choice determines a branch, $\beta(\kappa)$, of $\Phi$ and a branch of $S$ in a neighborhood of $\left(t\left(z^{0}\right), z^{0}\right)$. The branch $\beta(\kappa)$ gives a section of $\mathscr{S}(\kappa)$ for all $\kappa$.

There exist local coordinates $u_{1}, \ldots, u_{k}$ in $\mathscr{U}_{k, n}(z)$ centered at $t(z)$ such that the coordinates holomorphically depend on $z$ in a neighborhood of $z^{0}$ and

$$
S(t(u), z)=-i\left(u_{1}^{2}+\cdots+u_{n}^{2}\right)+g(z)
$$

for some function $g(z)$. For these coordinates and a small $\varepsilon>0$, denote by $d(z)$ the $\operatorname{disc}\left\{(u, z) \in \mathscr{U}_{k, n}(z) \mid u_{1}, \ldots, u_{k} \in \mathbb{R}, u_{1}^{2}+\cdots+u_{k}^{2} \leqq \varepsilon\right\}$.

The homology class of the cycle

$$
\delta(z, \kappa)=\left(d(z),\left.\beta(\kappa)\right|_{d(z)}\right)
$$


gives a generator of $H_{k}\left(B(z), B_{-}(z), \mathscr{S}(\kappa)\right)$. The classes $[\delta(z, \kappa)]$ form a flat section of the Gauss-Manin connection of the vector bundle over a neighborhood of $z^{0}$ with fiber $H_{k}\left(B(z), B_{-}(z), \mathscr{S}(\kappa)\right)$.

Set

$$
\Psi(z, \kappa)=(2 \pi \kappa)^{-\frac{k}{2}} \int_{\delta(z, \kappa)} N
$$

(3.3.7). Theorem [RV]. Let $\kappa=i s, s \in \mathbb{R}$, and $s \rightarrow+0$.

1. Then the function $\Psi(z)$ has an asymptotic expansion

$$
\Psi(z, i s) \sim \exp (-i S(t(z), z) / s) \sum_{j=0}^{\infty} g_{j}(z) s^{\prime}
$$

where $\left\{g_{j}\right\}$ are $\operatorname{Sing} L_{i_{i}}$-valued holomorphic functions defined in a neighborhood of $z^{0}$.

2. The function $\Psi(z, \kappa)$ gives a quasiclassical asymptotic solution to the $K Z$ equation in the sense of (2.5).

$$
g_{o}(z)=\operatorname{Hess}_{t}(-S(t(z), z))^{-\frac{1}{2}} \sum_{f_{K} \in L_{j}} A_{K}(t(z), z) f_{K}
$$

(3.3.8). Remark. Let $\delta^{0}(z, \kappa)$ be a singular chain in $B(z)$ with coefficients in $\mathscr{S}(\kappa)$ such that the boundary of $\delta^{\prime}(z, \kappa)$ lies in $B_{-}(z)$ and the class of $\delta^{\prime}(z, \kappa)$ in $H_{k}\left(B(z), B_{-}(z), \mathscr{S}(\kappa)\right)$ coincides with $[\delta(z, \kappa)]$. Then the function

$$
\Psi^{0}(z, \kappa)=(2 \pi \kappa)^{-\frac{k}{2}} \int_{\delta^{0}(z, \kappa)} N
$$

has the same asymptotic expansion as the function $\Psi(z, \kappa)$.

The vector

$$
g(t(z), z)=\sum_{f_{K} \in L_{\lambda}} A_{K}(t(z), z) f_{k}
$$

is called the Bethe vector, see [B, Ba, BF, FFR, G, R, TV].

It is shown in [V2], that

$$
B(g(t(z), z), g(t(z), z))=\operatorname{Hess}_{t}(S(t(z), z)),
$$

where $B$ is the Shapovalov form on $\operatorname{Sing} L_{i}$.

The group of permutations of coordinates $t_{1}, \ldots, t_{k}$ acts on the set of $t$-critical points of $S$. The Bethe vectors corresponding to $t$-critical points of the same orbit are identical. If two critical points $t=t^{1}(z)$ and $t=t^{2}(z)$ lie in different orbits, then the corresponding Bethe vectors are orthogonal with respect to the Shapovalov form,

$$
B\left(g\left(t^{1}(z), z\right), g\left(t^{2}(z), z\right)\right)=0 .
$$

Moreover, for generic $z \in \mathscr{U}_{n}$, there are exactly $\operatorname{dim} \operatorname{Sing} L_{\lambda}$ different orbits of nondegenerate $t$-critical points, and, consequently, the corresponding Bethe vectors form a basis in $\operatorname{Sing} L_{\lambda}$, see [RV]. 
(3.4). Homology Class Sitting on a Critical Point. Under the assumptions of Sect. (3.3), assume that for almost all $\kappa$ a homology class $[\gamma(\kappa)] \in H_{k}\left(\mathscr{U}_{k, n}\left(z^{0}\right)\right.$, $\mathscr{S}(\kappa))$ is given. Assume that a chain

$$
\gamma(\kappa)=\sum_{j=1}^{M}\left(c_{j}, \alpha_{j}(\kappa)\right)
$$

is given, such that:

For every $j, c_{j} \subset \mathscr{U}_{k, n}\left(z^{0}\right)$ is a singular cell.

For every $j, \alpha_{j}(\kappa)$ is a section of $\mathscr{S}(\kappa)$ over $c_{j}$ which has the following form:

Fix a branch of arguments of all functions $t_{j}-t_{l}, t_{j}-z_{l}, z_{l}-z_{m}$ for all $l, m$ in a neighborhood of $c_{j}$. This choice determines a branch $\beta_{j}(\kappa)$ of $\Phi$ over $c_{j}$. Then $\alpha_{j}(\kappa)=\varepsilon_{j}(\kappa) \beta_{j}(\kappa)$, where $\varepsilon_{j}(\kappa)$ is a rational function of $q(\kappa)^{\frac{1}{4}}$.

If $\kappa \in \mathbb{C}$ is such that $\varepsilon_{1}(\kappa), \ldots, \varepsilon_{m}(\kappa)$ are defined, then the chain $\gamma(\kappa)$ is a cycle representing $[\gamma(\kappa)]$.

In this case we will say that the class $[\gamma(\kappa)]$ is flat with respect to $\kappa$, and $\gamma(\kappa)$ is a flat chain representative.

Assume that $[\gamma(\kappa)]$ is flat. Assume that $[\gamma(\kappa)]$ has a representing chain $\gamma(\kappa)$ of the form (3.4.1)-(3.4.4) and such that

$\left(c_{1}, \alpha_{1}(\kappa)\right)$ has the form $\left(d\left(z^{0}\right),\left.\varepsilon_{1}(\kappa) \beta(\kappa)\right|_{d\left(z^{0}\right)}\right)$, where $\left(d\left(z^{0}\right),\left.\beta(\kappa)\right|_{d\left(z^{0}\right)}\right)$ is described in (3.3.5) and $\varepsilon_{1}(\kappa)$ is a rational function of $q(\kappa)^{\frac{1}{4}}$.

For $\kappa=i s, s \in \mathbb{R}_{>0}$, and for every $j=2, \ldots, M$, we have

$$
\lim _{s \rightarrow+0} \sup _{c_{j}}\left|\alpha_{j}(\kappa)\right| /\left|\varepsilon_{1}(\kappa) \beta(\kappa)\left(t\left(z^{0}\right), z^{0}\right)\right|=0 .
$$

In this case we will say that the class $[\gamma(\kappa)]$ sits on the t-critical point $\left(t\left(z^{0}\right), z^{0}\right)$.

Assume that $[\gamma(\kappa)]$ sits on $\left(t\left(z^{0}\right), z^{0}\right)$. Let $[\gamma(\kappa)] \in H_{k}\left(\mathscr{U}_{k, n}(z), \mathscr{S}(\kappa)\right)$ be the class such that for a fixed $\kappa$ the map $z \mapsto[\gamma(\kappa)]$ forms a flat section of the GaussManin connection over a neighborhood of $z^{0}$ and $\left[\gamma\left(z^{0}, \kappa\right)\right]=[\gamma(\kappa)]$.

(3.4.7). Theorem. Under the above conditions consider the $\operatorname{Sing} L_{\lambda}$-valued function

$$
\Psi(z, \kappa)=\int_{[\gamma(z, \kappa)]} N
$$

which for a fixed $\kappa$ gives a solution to the $K Z$ equation with parameter $\kappa$. Assume that $\kappa=i s, s \in \mathbb{R}$, and $s \rightarrow+0$. Then

1. The function $\Psi$ has an asymptotic expansion

$$
\Psi(z, i s) \sim F(\kappa) \exp (-i S(t(z), z) / s) \sum_{j=0}^{\infty} g_{j}(z) s^{j},
$$

where $\left\{g_{j}\right\}$ are $\operatorname{Sing} L_{\lambda^{-}}$-valued holomorphic functions defined in a neighborhood of $z^{0}$. 
2. The function $\Psi$ gives a quasiclassical aysmptotic solution to the $K Z$ equation in the sense of (2.5).

3. $g_{0}(z)$ is given by (3.3.7.3).

The theorem is a corollary of (3.3.7).

Consider the natural action of $S_{k}$ on singular chains and homology classes. Let $[\gamma(z, \kappa)]$ be as above. Define a new skew symmetric class $[\gamma(z, \kappa)]_{-} \in H_{k}\left(\mathscr{U}_{k, n}(z)\right.$, $\mathscr{S}(\kappa))_{-}$by

$$
[\gamma(z, \kappa)]_{-}=\sum_{\sigma \in S_{k}}(-1)^{|\sigma|} \sigma[\gamma(z, \kappa)]
$$

We have

$$
\int_{[\gamma(z, \kappa)]_{-}} N=k ! \int_{[\gamma(z, \kappa)]} N
$$

(3.4.10). Corollary. The intergral in (3.4.9) has an asymptotic expansion as $\kappa=i$, $s \in \mathbb{R}$, and $s \rightarrow+0$. The asymptotic expansion is equal to the asymptotic expansion in (3.4.7.1) multiplied by $k$ !.

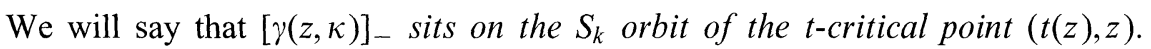
(3.5). Example of a Bethe Vector. Let

$$
\begin{aligned}
\Phi_{k, m_{1}, m_{2}, \kappa}(t) & =\prod_{j=1}^{k}\left(-t_{J}\right)^{-\frac{m_{1}}{\kappa}}\left(1-t_{J}\right)^{-\frac{m_{2}}{\kappa}} \cdot \prod_{1 \leqq j<l \leqq k}\left(t_{j}-t_{l}\right)^{2 / \kappa}, \\
S(t) & =S_{k, m_{1}, m_{2}}(t, \kappa)=\kappa \ln \Phi(t) .
\end{aligned}
$$

(3.5.2). Theorem, [V2], cf. [Sz, Sect. 6.7].

1. If $t^{0}=\left(t_{1}, \ldots, t_{k}\right)$ is a critical point of $\Phi$, then the symmetric functions

$$
\lambda_{1}=t_{1}+\cdots+t_{k}, \quad \lambda_{2}=\sum t_{1} t_{j}, \quad \ldots, \quad \lambda_{k}=t_{1} \cdot \cdots \cdot t_{k}
$$

are given by

$$
\lambda_{l}=\left(\begin{array}{l}
k \\
l
\end{array}\right) \prod_{j=1}^{l} \frac{\left(m_{1}+j-k\right)}{\left(m_{1}+m_{2}+j+1-2 k\right)}
$$

for all $l$.

2. The Bethe vector is $\operatorname{Sing}\left(L\left(m_{1}\right) \otimes L\left(m_{2}\right)\right)_{m_{1}+m_{2}-2 k}$ corresponding to the critical point $t^{0}$ is given by

$$
\sum_{p=0}^{k}(-1)^{p}\left(\begin{array}{c}
k \\
p
\end{array}\right) \frac{\prod_{j=0}^{k-1}\left(m_{1}+m_{2}-2 k+j+2\right)}{\prod_{j=0}^{p-1}\left(m_{1}-j\right) \prod_{j=0}^{k-p-1}\left(m_{2}-j\right)} f^{p} v_{1} \otimes f^{k-p} v_{2} .
$$

3.

$$
\operatorname{Hess}\left(S\left(t^{0}\right)\right)=k ! \prod_{j=0}^{k-1} \frac{\left(m_{1}+m_{2}-2 k+j+2\right)^{3}}{\left(m_{1}-j\right)\left(m_{2}-j\right)}
$$

(3.6). Asymptotics of Critical Points and Bethe Vectors. In (2.2) we have constructed asymptotic zones. In this section we will describe asymptotics of $t$-critical points and Bethe vectors in an asymptotic zone, cf. [RV]. 
Asymptotic zones are numerated by $n$-trees and elements of the symmetric group $S_{n}$. We will consider the case of the identity permutation. The case of an arbitrary permutation is treated similarly.

For an $n$-tree $T$ consider the asymptotic zone

$$
u_{T, \text { id }}=\left\{z_{1}+\cdots+z_{n},\left\{u_{w}, T, \text { id }\right\}_{w \in \operatorname{In}_{T}}\right\}: D_{\text {id }} \rightarrow \mathbb{R} \times\left(\mathbb{R}_{>0}\right)^{n-1} .
$$

Consider the function $\Phi(t, z)$ defined by (3.1.1).

Mark the tops of $T$ by $m_{1}, \ldots, m_{n}$. Let the set Adm be the set of all admissible colorings of $T$ having weight $\lambda=m_{1}+\cdots+m_{n}-2 k$, see (1.2).

(3.6.2). Theorem. Assume that $z \in D_{\mathrm{id}}$ and $u_{w, T, \mathrm{dd}}(z) \ll 1$ for all $w \in \operatorname{In}_{T}$. Then we have the following three statements.

1. The number of $S_{k}$ orbits of nondegenerate t-critical points of $\Phi(t, z)$ is equal to $\operatorname{dim} \operatorname{Sing} L_{\lambda}$, see [RV].

2. The $S_{k}$ orbits of nondegenerate t-critical points can be numerated by elements of Adm in such a way that the $S_{k}$-orbit corresponding to a coloring $c \in \mathrm{Adm}$ has a t-critical point $\left(t^{c}(z), z\right)$ of the following form:

Let $\left\{M_{w}\right\}_{w \in \operatorname{In}_{T}}$ be an arbitrary partition of $\{1, \ldots, k\}$ into a union of disjoint subsets such that $\# M_{w}=c(w)$. For each $M_{w}$, fix its arbitrary ordering $M_{w}=$ $\left\{j_{1}(w), \ldots, j_{c(w)}(w)\right\}$. For any $w \in \operatorname{In}_{T}$ define a number $l(w)$ as follows. The tops of the tree $T$ are numerated by $1, \ldots, n$ from left to right. The number $l(w)$ is the maximum of the indices of the tops lying on the left branch of the tree $T$ at $w$. Let $m^{l e}(w)$ and $m^{r}(w)$ be the numbers defined in (1.2) for a vertex $w$ of a colored tree $T$ with marked tops. Consider the function $S_{c(w), m^{l e}(w), m^{r}(w)}$ defined by (3.5.1). Let $\left(a_{1}(w), \ldots, a_{c(w)}(w)\right)$ be coordinates of a critical point of the function $S_{c(w), m^{l e}(w), m^{r}(w)}$. Then the critical point $\left(t^{c}(z), z\right)$ has the form:

$$
t_{J p(w)}^{c}(z)-z_{l(w)}=\left(a_{p}(w)+\mathcal{O}\left(u_{T, \mathrm{dd}}(z)\right)\right) \prod_{y} u_{y, T, 1 \mathrm{~d}} .
$$

The product is over all internal vertices $y$ lying on the shortest path in T connecting $w$ and the root of $T$ (the vertex $w$ is included). The function $\mathcal{O}$ is a function of $u_{x, T, \mathrm{id}}, x \in \ln _{T}$, holomorphic in a neighborhood of the set $\left\{u_{x, T, \mathrm{~d}}=0, x \in \operatorname{In}_{T}\right\}$, and such that $\mathcal{O}(u=0)=0$.

3. The Bethe vector corresponding to $\left(t^{c}(z), z\right)$ has the following form:

$$
g\left(t^{c}(z), z\right)=(v(c, 0, T, \mathrm{id})+\mathcal{O}(u(z))) \prod_{w \in \operatorname{In}_{T}}\left(u_{w, T, \mathrm{id}}\right)^{-c(w)-b(w)},
$$

where $b(w)=\sum_{y} c(y)$, the sum is over $y \in \operatorname{In}_{T}$ such that $y$ lies on the right or on the left branch of $T$ at $w$. The function $\mathcal{O}(u)$ has the same structure as in (3.6.2.2).

To prove the theorem we consider new variables $\left\{x_{i_{p}(w)}, u_{T, \mathrm{dd}}\right\}$ defined by

$$
\begin{aligned}
t_{j p(w)}-z_{l(w)} & =x_{j p}(w) \prod_{y} u_{y, T, \mathrm{id}} . \\
z_{m} & =z_{m}\left(u_{T, \mathrm{id}}\right)
\end{aligned}
$$


where the product is the same as in (3.6.2.2), and the second formula is determined by $(3.6 .1)$. It is easy to see that

$$
\begin{aligned}
\Phi(t(x, u), z(u))=(-1)^{A / \kappa} \prod_{w \in \operatorname{In}_{T}}\left(u_{w, T, \mathrm{id}}\right)^{\mu(c, T, \mathrm{dd}, w) / \kappa} \\
\\
\cdot\left(\prod_{w \in \operatorname{In}_{T}} \Phi_{c(w), m^{l}(w), m^{r}(w), k}\left(\left\{x_{j_{p}(w)}\right\}\right)+\mathcal{O}(u)\right),
\end{aligned}
$$

where $A$ is some constant, $\{\mu(c, T, i d, w)\}$ are the eigenvalues of the vector $v(c, 0, T, i d)$ with respect to the operators $\left\{\Omega_{w, T, \mathrm{dd}}\right\}$, see $(2.3 .1), \mathcal{O}$ is a function of $x, u$ holomorphic in a neighborhood of the set

$$
X=\left\{x_{j_{p}(w)}=a_{p}(w), u_{w, T, \mathrm{~d}}=0 \text { for all } w, p\right\},
$$

and which is zero on $X$. This statement implies (3.6.2.2) and (3.6.2.3). Statement (3.6.2.1) is proved in [RV].

(3.6.6) Remark. There are integral representations for solutions to the $\mathrm{KZ}$ equation with values in a tensor product of modules over an arbitrary Kac-Moody Lie algebra $\mathfrak{g}$, see [SV, V1]. There are obvious $\mathfrak{g}$ analogs of (3.3.7), (3.4.4) and (3.6.2), cf. [RV].

\section{Integral Representations for Solutions to the $\mathrm{KZ}$ Equation and the Quantum Group $U_{q}$}

(4.1). Complex $\left[V 1\right.$, Sect. 5]. For $\kappa \in \mathbb{C}$, consider the $\mathbb{C}$-algebra $U_{q=q(\kappa)}$. For $k>0$, let

$$
\left(U_{q=q(\kappa)}^{-}\right)_{k}=\mathbb{C} f^{k} \subset U_{q=q(\kappa)} \quad \text { and } \quad U_{q=q(\kappa)}^{-}=\bigoplus_{k>0} \mathbb{C} f^{k}
$$

The map $\mu: U^{-} \rightarrow U^{-} \otimes U^{-}$,

$$
\mu: f^{k} \mapsto \sum_{l=1}^{k-1}\left(\begin{array}{l}
k \\
l
\end{array}\right)_{q(k)} f^{l} \otimes f^{k-l}
$$

defines a coalgebra structure on $U^{-}$, here

$$
\left(\begin{array}{l}
k \\
l
\end{array}\right)_{q(\kappa)}=\frac{(k)_{q(\kappa)} !}{(l)_{q(\kappa)} !(k-l)_{q(\kappa)} !}
$$

is the $q$-binomial coefficient.

For a $U_{q=q(\kappa)}$-module $M$ with highest weights, the map $v: M \rightarrow U_{q=q(\kappa)}^{-} \otimes M$,

$$
v: u \mapsto \sum_{l>0} f^{l} \otimes \frac{\left(q^{-\frac{h}{4}} e\right)^{l}}{[l]_{q(\kappa)} !} u
$$

defines a $U_{q=q(\kappa)}^{-}$-comodule structure on $M$.

For $k \geqq 0$, set

$$
C^{k}(M)=\left(U_{q=q(\kappa)}^{-}\right)^{\otimes k} \otimes M
$$


Define $d: C^{k} \rightarrow C^{k+1}$ by

$$
\begin{aligned}
d: a_{k} \otimes \cdots \otimes a_{1} \otimes u \mapsto & -a_{k} \otimes \cdots \otimes a_{1} \otimes v(u) \\
& +\sum_{l=1}^{k}(-1)^{l+1} a_{k} \otimes \cdots \otimes \mu\left(a_{l}\right) \otimes \cdots \otimes a_{1} \otimes u .
\end{aligned}
$$

For any $\lambda$ and $k$, set

$$
C_{i}^{k}(M)=\underset{l_{1}, \ldots, l_{k}}{\bigoplus}\left(U^{-}\right)_{l_{1}} \otimes \cdots \otimes\left(U^{-}\right)_{l_{k}} \otimes M_{\lambda-l_{1}-\cdots-l_{k}}
$$

Then $C^{k}=\bigoplus_{i} C_{i}^{k}$. The differential $d$ preserves the grading. For any $\lambda$ we have a complex $\left(C_{\lambda}^{\bullet}(M), d\right)$ and

$$
H^{0}\left(C_{i}^{\bullet}, d\right)=\operatorname{ker} e \cap M_{\lambda}
$$

for a nonrational $\kappa$. Assume that $\kappa$ is not a rational number.

For an integer $m$, let $V(m, q=q(\kappa))$ (resp. $L(m, q=q(\kappa))$ be the $U_{q=q(\kappa)}$ Verma module (resp. irreducible module) with highest weight $m$.

For any $\sigma \in S_{n}, \lambda \in \mathbb{Z}$, and natural $m_{1}, \ldots, m_{n}$, set

$$
\begin{gathered}
\left.V^{\sigma}(q=q(\kappa))=V\left(m_{\sigma(1), q}=q(\kappa)\right)\right) \otimes \cdots \otimes V\left(m_{\sigma(n), q}=q(\kappa)\right), \\
\operatorname{Sing} V^{\sigma}(q=q(\kappa))_{\lambda}=\left(V^{\sigma}\right)_{i} \cup \operatorname{ker} e .
\end{gathered}
$$

Let $L^{\sigma}(q=q(\kappa))$ and $\operatorname{Sing} L^{\sigma}(q=q(\kappa))$, be the corresponding objects constructed from the irreducible modules, Denote by

$$
\text { pr: } \operatorname{Sing} V^{\sigma}(q=q(\kappa))_{\lambda} \rightarrow \operatorname{Sing} L^{\sigma}(q=q(\kappa))_{\lambda}
$$

the natural epimorphism.

For any $j=1, \ldots, n-1$, we have an isomorphism

$$
R_{j, j+1}:\left(U^{-}\right)^{\otimes k} \otimes V^{\sigma} \rightarrow\left(U^{--}\right)^{\otimes k} \otimes V^{\sigma \tau},
$$

where $\tau \in S_{n}$ is the transposition of $j$ and $(j+1) . R_{j, j+1}$ commutes with the differential and induces an isomorphism of cohomology groups of the corresponding complexes.

(4.2). The Main Result. Assume that $\kappa \in \mathbb{C}$ is not a rational number. Consider the function $\Phi(t, z)$ defined by (3.1.1) and the objects associated with the function $\Phi(t, z)$ in (3.1) and (3.2).

For $z \in \mathscr{U}_{n}$, let $\mathscr{C}_{\bullet}\left(\mathscr{U}_{k, n}(z), \mathscr{S}(\kappa)\right)$ be the complex of singular chains in $\mathscr{U}_{k, n}(z)$ with coefficients in $\mathscr{S}(\kappa)$. The group $S_{k}$ of permutations of corrdinates $t_{1}, \ldots, t_{k}$ acts on $\mathscr{C}_{\bullet}$. Denote by $\mathscr{C}_{\bullet}\left(\mathscr{U}_{k, n}(z), \mathscr{S}(\kappa)\right)_{\text {- }}$ the skew symmetric part of the action.

(4.2.1). Theorem. For any $\sigma \in S_{n}$ and any $z \in D_{\sigma}$ there exists a monomorphism of complexes

$$
v(z, \kappa): C_{\lambda}^{\bullet}\left(V^{\sigma}(q=q(\kappa))\right) \rightarrow C_{k-\bullet}\left(\mathscr{U}_{k, n}(z), \mathscr{S}(\kappa)\right)_{-},
$$


where $\lambda=m_{1}+\cdots+m_{n}-2 k$. Denote the image of $v$ by

$$
C_{\bullet}\left(\mathscr{U}_{k, n}(z), \mathscr{S}(\kappa)\right)_{-} \subset \mathscr{C}_{\bullet}\left(\mathscr{U}_{k, n}(z), \mathscr{S}(\kappa)\right)_{-} .
$$

The monomorphism has the following six properties:

1. $v(z, \kappa)$ is a quasiisomorphism. Denote by the same symbol $v(z, \kappa)$ the induced isomorphism of homology groups: $H^{\bullet}\left(C_{\lambda}^{\bullet}\left(V^{\sigma}(q=q(\kappa))\right), d\right) \cong H_{k-\bullet}\left(\mathscr{U}_{k, n}(z)\right.$, $\mathscr{S}(\kappa))_{-}$.

2. $v(z, \kappa)$ is flat with respect to the Gauss-Manin connection. Namely, if

$$
P\left(z^{1}, z^{2}\right): H_{\bullet}\left(\mathscr{U}_{k, n}\left(z^{1}\right), \mathscr{S}(\kappa)\right)_{-} \rightarrow H_{\bullet}\left(\mathscr{U}_{k, n}\left(z^{2}\right), \mathscr{S}(x)\right)_{-}
$$

is the isomorphism of the Gauss-Manin connection along a curve in $D_{\sigma}$ from a point $z^{1} \in D_{\sigma}$ to a point $z^{2} \in D_{\sigma}$, then $v\left(z^{2}, \kappa\right)=P\left(z^{1}, z^{2}\right) \cdot v\left(z^{1}, \kappa\right)$.

3. Let $\tau$ be the transposition of $j$ and $j+1$ for $j=1, \ldots, n-1$. Connect the domains $D_{\sigma}$ and $D_{\sigma \tau}$ by a curve $\gamma:[0,1] \rightarrow \mathscr{U}_{n}$ of the following form. Let $\left(z_{1}^{0}, \ldots, z_{n}^{0}\right) \in$ $D_{\sigma}, z_{\sigma(1)}^{0}<\cdots<z_{\sigma(n)}^{0}$. Assume that $z_{\sigma(j)}$ and $z_{\sigma(j+1)}$ are very close. Set $z_{l}(\gamma(s))=$ $z_{l}^{0}$ for $l \neq \sigma(j+1)$, set $z_{\sigma(j+1)}(\gamma(s))=\exp (\pi i s)\left(z_{\sigma(j+1)}^{0}-z_{\sigma(j)}^{0}\right)+z_{\sigma(j)}^{0}$. Let

$$
P_{\gamma}: H_{\bullet}\left(U_{k, n}(\gamma(0)), \mathscr{S}(\kappa)\right)_{-} \rightarrow H_{\bullet}\left(\mathscr{U}_{k, n}(\gamma(1)), \mathscr{S}(\kappa)\right)_{-}
$$

be the isomorphism of the Gauss-Manin connection along $\gamma$. Let

$$
R_{j, j+1}: H^{\bullet}\left(C_{i}^{\bullet}\left(V^{\sigma}(q=q(\kappa)), d\right)\right) \rightarrow H^{\bullet}\left(C_{\lambda}^{\bullet}\left(V^{\sigma \tau}(q=q(\kappa)), d\right)\right)
$$

be the R-matrix isomorphism. Then $P_{\gamma} \cdot v(\gamma(0), \kappa)=v(\gamma(1), \kappa) \cdot R_{j, j+1}$.

4. Consider the $K Z$ equation with parameter $\kappa$ and with values in $\operatorname{Sing} L_{\lambda}$, see (3.2). Let $\operatorname{Sol}(\kappa)_{\lambda, \sigma}$ be the space of solutions to the $K Z$ equation over $D_{\sigma}$. By (4.2.1.1) and (4.2.1.2), for any $v \in \operatorname{Sing} V^{\sigma}(q=q(\kappa))_{\lambda}$, the function

$$
\psi_{v}: D_{\sigma} \rightarrow \operatorname{Sing} L_{\lambda}, \quad z \mapsto \int_{v(z, \kappa) v} N
$$

is a solution to the $K Z$ equation. Here $N$ is defined by (3.2.3). Hence, by (4.2.1.1) and (4.2.1.2), we have a homomorphism

$$
\mathscr{V}_{\sigma}(\kappa): \operatorname{Sing} V^{\sigma}(q=q(\kappa))_{\lambda} \rightarrow \operatorname{Sol}(\kappa)_{\lambda, \sigma} .
$$

Let pr: $\operatorname{Sing} V^{\sigma}(q=q(\kappa))_{\lambda} \rightarrow \operatorname{Sing} L^{\sigma}(q=q(\kappa))_{\lambda}$ be the canonical epimorphism. We claim that $\operatorname{ker} p r \subset \operatorname{ker} \mathscr{V}_{\sigma}(\kappa)$. Hence we have a homomorphism

$$
\pi_{\sigma}(\kappa): \operatorname{Sing} L^{\sigma}(q=q(\kappa))_{\lambda} \rightarrow \operatorname{Sol}(\kappa)_{\lambda, \sigma} .
$$

5. For an n-tree $T$, let $\mathscr{B}_{T, \sigma}(\kappa)=\{v(c, 0, T, \sigma, \kappa)\}$ be the basis in $\operatorname{Sing} L^{\sigma}(q=$ $q(\kappa))_{\lambda}$ corresponding to the tree $T$ with tops marked by $m_{\sigma}(1), \ldots, m_{\sigma}(n)$, see (1.2). Then for any vector $v \in \mathscr{B}_{T, \sigma}(\kappa)$ we have

$$
\pi_{\sigma}(\kappa)(v(c, 0, T, \sigma, \kappa))=\psi_{c, T, \sigma} .
$$

Here $\psi_{c, T, \sigma}$ is the asymptotic solution defined by (2.3.1).

Corollary. $\pi_{\sigma}(\kappa)$ is an isomorphism.

6. Let $u_{T, \sigma}$ be the asymptotic zone constructed in (2.2). If $z \in D_{\sigma}$ and $\left\{u_{w, T, \sigma}(z)\right\}_{w \in \operatorname{In}_{T}}$ are small enough, then for any $v(c, 0, T, \sigma, \kappa) \in \mathscr{B}_{T, \sigma}$ the class 
$v(z, \kappa) v(c, 0, T, \sigma, \kappa)$ sits on the $S_{k}$-orbit of the $t$-critical point $\left(t^{c}(z), z\right)$ corresponding to the coloring $c$, see (3.6.2). Moreover, the asymptotic expansion (3.4.10), (3.4.7.1), and (3.3.7.1) of $\pi_{\sigma}(\kappa) v(c, 0, T, \sigma, \kappa)$ has the form (2.5.8) and properties described in (2.5.7) and (2.5.9).

Theorem (4.2.1) implies (2.4.1), (2.5.7), and (2.5.9). Theorem (4.2.1) is proved in $(4.4)-(4.6)$.

(4.3). Another Form of $U_{q} s l_{2}$. Define the $\mathbb{Q}\left(q^{\frac{1}{4}}\right)$-algebra $\widetilde{U}_{q}$ as the algebra generated by the symbols $E, F, H$ with the relations

$$
\begin{aligned}
& {[H, E]=2 E,} \\
& {[H, F]=-2 F,} \\
& {[E, F]=q^{H / 2}-q^{-H / 2} .}
\end{aligned}
$$

$\widetilde{U}_{q}$ has a Hopf algebra structure with the comultiplication $\Delta$ defined by

$$
\begin{aligned}
& \Delta(H)=H \otimes 1+1 \otimes H, \\
& \Delta(E)=E \otimes q^{H / 4}+q^{-H / 4} \otimes E, \\
& \Delta(F)=F \otimes q^{H / 4}+q^{-H / 4} \otimes F .
\end{aligned}
$$

Let $\widetilde{U}_{q=q(\kappa)}$ be the $\mathbb{C}$-algebra obtained from $\widetilde{U}_{q}$ by specializing the indeterminate $q^{1 / 4}$ to $q(\kappa / 4)$.

For $k>0$, set $\left(\widetilde{U}^{-}\right)_{k}=\mathbb{C} F^{k}$ and $\widetilde{U}^{-}=\bigoplus_{k>0} \mathbb{C} F^{k}$. The map $\tilde{\mu}: \widetilde{U}^{-} \rightarrow \widetilde{U}^{-} \otimes$ $\widetilde{U}^{-}$given by (4.1.1), in which $f$ is replaced by $F$, defines a coalgebra structure. For a $\widetilde{U}_{q=q(\kappa)}$-module $\tilde{M}$ with highest weights, the map

$$
\tilde{v}: u \mapsto \sum_{l>0} F^{l} \otimes E^{(l)} u
$$

defines a $\widetilde{U}^{-}$-comodule structure on $\tilde{M}$. For any $\lambda$ define a complex $\left(\widetilde{C}_{\lambda}^{\bullet}(\tilde{M}), \tilde{d}\right)$ as in $(4.1)$.

The Hopf algebras $U_{q}$ and $\widetilde{U}_{q}$ are isomorphic, an isomorphism $\pi: U_{q} \rightarrow \widetilde{U}_{q}$ is given by $\pi(h)=H, \pi(e)=q^{H / 4} E, \pi(f)=F q^{-H / 4}$. In particular, these formulae give an isomorphism of $U_{q=q(\kappa)}$ and $\widetilde{U}_{q=q(\kappa)}$.

Assume that $\kappa$ is not a rational number.

For an integral $m$, let $V(m, q=q(\kappa))$ be the $U_{q=q(\kappa)}$ Verma module with the highest weight $m$, the generating vector $v_{m}$, and the basis $f^{(l)} v_{m}, l \geqq 0$. Let $\widetilde{V}(m, q=q(\kappa)), \tilde{v}_{m}, F^{(l)} \tilde{v}_{m}$ be the corresponding objects over $\widetilde{U}_{q=q(\kappa)}$. Let

$$
\pi_{m}: V(m, q=q(\kappa)) \rightarrow \widetilde{V}(m, q=q(\kappa)), \quad f^{(l)} v_{m} \mapsto \frac{\left(F q^{-H / 4}\right)^{l}}{[l]_{q(\kappa)} !} \tilde{v}_{m} .
$$

Then for any $a \in U_{q=q(\kappa)}$ and $u \in V(m, q=q(\kappa))$ we have $\pi_{m}(a u)=\pi(a) \pi_{m}(u)$.

For natural $m_{1}, \ldots, m_{n}$ and $\sigma \in S_{n}$, let

$$
V^{\sigma}(q=q(\kappa))=V\left(m_{\sigma(1)}, q=q(\kappa)\right) \otimes \cdots \otimes V\left(m_{\sigma(n)}, q=q(\kappa)\right)
$$


and

$$
\widetilde{V}^{\sigma}(q=q(\kappa))=\widetilde{V}\left(m_{\sigma(1)}, q=q(\kappa)\right) \otimes \cdots \otimes \widetilde{V}\left(m_{\sigma(n)}, q=q(\kappa)\right) .
$$

For any $k$ and $\lambda$, define a map

$$
\begin{gathered}
\Psi: C_{\lambda}^{k}\left(V^{\sigma}(q=q(\kappa))\right) \rightarrow \widetilde{C}_{\lambda}^{k}\left(\widetilde{V}^{\sigma}(q=q(\kappa))\right), \\
f^{l_{1}} \otimes \cdots \otimes f^{l_{k}} \otimes u_{1} \otimes \cdots \otimes u_{n} \mapsto F^{l_{1}} \otimes \cdots \otimes F^{l_{k}} \otimes \pi_{m_{\sigma(1)}}\left(u_{1}\right) \otimes \cdots \otimes \pi_{m_{\sigma(n)}}\left(u_{n}\right) .
\end{gathered}
$$

Then

$\Psi$ defines an isomorphism of $C_{i}^{\bullet}\left(V^{\sigma}(q=q(\kappa))\right)$ and $\widetilde{C}_{i}^{\bullet}\left(\widetilde{V}^{\sigma}(q=q(\kappa))\right)$.

For a nonnegative integer $m$ denote by $\widetilde{L}(m, q=q(\kappa))$ the irreducible $\widetilde{U}_{q=q(\kappa)^{-}}$ module with highest weight $m$. Set

$$
\begin{aligned}
& \text { Sing } \widetilde{V}(q=q(\kappa))_{i}=\left\{v \in \widetilde{V}^{\sigma}(q=q(\kappa)) \mid q^{H} v=q^{\lambda}(\kappa) v, E v=0\right\}, \\
& \operatorname{Sing} \widetilde{L}^{\sigma}(q=q(\kappa))_{\lambda}=\left\{v \in \widetilde{L}^{\sigma}(q=q(\kappa)) \mid q^{H} v=q^{\lambda}(\kappa) v, E v=0\right\} .
\end{aligned}
$$

(4.4). Construction of the Monomorphism v. Assume that $\kappa, m_{1}, \ldots, m_{n}, \lambda, k$ are the same as in (4.2).

In [V1] a monomorphism

$$
\left.\tilde{v}(z, \kappa): \widetilde{C}_{\lambda}^{\bullet} \widetilde{V}^{\sigma}(q=q(\kappa))\right) \rightarrow \mathscr{C}_{k-\bullet}\left(\mathscr{U}_{k, n}(z), \mathscr{S}(\kappa)\right)_{-}
$$

is constructed for any $\sigma \in S_{n}$ and any $z \in D_{\sigma}$. Denote the image of the monomorphism by $\widetilde{C}_{\bullet}\left(\mathscr{U}_{k, n}(z), \mathscr{S}(\kappa)\right)_{-} \subset \mathscr{C}_{\bullet}\left(\mathscr{U}_{k, n}(z), \mathscr{S}(\kappa)\right)_{-}$. It is proved in [V1] that the monomorphism has the following four properties:

$\tilde{v}(z, \kappa)$ is a quasiisomorphism, cf. (4.2.1.1) and [V1, Sect. 8.2]. Denote by the same symbol $\tilde{v}(z, \kappa)$ the induced isomorphism in homology groups.

$\tilde{v}(z, \kappa)$ is flat with respect to the Gauss-Manin connection, cf. (4.2.1.2) and [V1, Sect. 8.2].

$\tilde{v}(z, \kappa)$ transforms the $R$-matrix action on $\widetilde{C}_{\lambda}^{\bullet}\left(\widetilde{V}^{\sigma}(q=q(\kappa))\right)$ into the monodromy action on $\widetilde{C}_{k-\bullet}\left(\mathscr{U}_{k, n}(z), \mathscr{S}(\kappa)\right)_{-}$, cf. the precise statement in (4.2.1.3) and [V1, Sect. 8.14].

For any $v \in \operatorname{Sing} \widetilde{V}^{\sigma}(q=q(\kappa))_{\lambda}$, the function

$$
\tilde{\psi}: D_{\sigma} \rightarrow \operatorname{Sing} L_{\lambda,}, \quad z \mapsto \int_{\tilde{v}(z, \kappa) v} N,
$$

is a solution to the $\mathrm{KZ}$ equation. By (4.4.3) we have a homomorphism

$$
\widetilde{\mathscr{V}}_{\sigma}(\kappa): \operatorname{Sing} \widetilde{V}(q=q(\kappa))_{\lambda} \rightarrow \operatorname{Sol}(\kappa)_{\lambda, \sigma} .
$$

Let $p r: \operatorname{Sing} \widetilde{V}(q=q(\kappa))_{\lambda} \rightarrow \operatorname{Sing} \widetilde{L}(q=q(\kappa))_{\lambda}$ be the canonical projection. It is proved in [V1, Sect. 12.2] that $\operatorname{ker} p r \subset \operatorname{ker} \widetilde{\mathscr{V}}$. Hence, we have a homomorphism

$$
\tilde{\pi}_{\sigma}: \operatorname{Sing} \widetilde{L}^{\sigma}(q=q(\kappa))_{\lambda} \rightarrow \operatorname{Sol}(\kappa)_{\lambda, \sigma} .
$$


Let $\Psi: C_{\lambda}^{k}\left(V^{\sigma}(q=q(\kappa))\right) \rightarrow \widetilde{C}_{\lambda}^{k}\left(\widetilde{V}^{\sigma}(q=q(\kappa))\right)$ be the isomorphism constructed in (4.3.4). We set

$$
v(z, \kappa)=(2 \pi \kappa)^{-\frac{k}{2}} \tilde{v}(z, \kappa) \Psi .
$$

Obviously, this monomorphism has properties (4.2.1.1)-(4.2.1.4). We will prove that the monomorphism has properties (4.2.1.5) and (4.2.1.6).

(4.5). Proof of Theorem (4.2.1) for $n=2$. For $n=2$ we have

$$
\Phi(t, z)=\left(z_{2}-z_{1}\right)^{m_{1} m_{2} / \kappa} \prod_{1 \leqq l<j \leqq \kappa}\left(t_{l}-t_{J}\right)^{2 / k} \prod_{l=1}^{k} \prod_{j=1}^{2}\left(z_{j}-t_{l}\right)^{-m_{j} / \kappa} .
$$

There are two permutations in $S_{2}$ : the identity permutation $i d$ and permutation $(2,1)$. We will prove properties (4.2.1.5) and (4.2.1.6) for $D_{\text {id }}$. There is only one 2-tree $T$, and therefore, there is only one asymptotic zone in $D_{\text {id }}$ :

$u_{T, \text { id }}=\left\{z_{1}+z_{2}, u=z_{2}-z_{1}\right\}$.

Introduce the following new coordinates $x_{1}, \ldots, x_{l}$ :

$$
t_{l}-z_{1}=u \cdot x_{l}, \quad l=1, \ldots, k
$$

cf. (3.6.3). Then

$$
\Phi(t(x, u), z(u))=(-1)^{m_{1} m_{2} / 2 \kappa} u^{\left(\frac{m_{1} m_{2}}{2}-k\left(m_{1}+m_{2}\right)+k(k-1)\right) / \kappa} \Phi_{k, m_{1}, m_{2}, k}(x),
$$

where the function $\Phi_{k, m_{1}, m_{2}, k}$ is given by (3.5.1).

Let $\Delta=\left\{x \in \mathbb{R}^{k} \mid 0<x_{1}<\cdots<x_{k}<1\right\}$.

Let $v_{m_{l}} \in L\left(m_{j}\right)$ be the generating vector of the $s l_{2}$ irreducible module. Let $\tilde{v}_{m_{j}} \in \widetilde{L}\left(m_{j}, q=q(\kappa)\right)$ be the generating vector of the $\widetilde{U}_{q=q(\kappa)}$ irreducible module. Let

$$
\begin{aligned}
v= & \sum_{p=0}^{k}(-1)^{p} \frac{\left(m_{2}-k+1\right)_{q(\kappa)} \ldots\left(m_{2}-k+p\right)_{q(k)}}{\left(m_{1}\right)_{q(\kappa)} \ldots\left(m_{1}-p+1\right)_{q(\kappa)}} \\
& \times q(\kappa)^{-p\left(m_{2}-2 k+p+1\right) / 2} F^{(p)} \tilde{v}_{m_{1}} \otimes F^{(k-p)} \tilde{v}_{m_{2}}
\end{aligned}
$$

be the generating vector of $\operatorname{Sing} \widetilde{L}_{i}^{\text {ld }}(q=q(\kappa))$. Set $\gamma(z)=\tilde{v}(z, \kappa) v \in$ $H_{k}\left(\mathscr{U}_{2, k}(z), \mathscr{P}(\kappa)\right)_{\text {- for }} z \in D_{1 \mathrm{~d}}$.

According to the explicit construction of $v(z, \kappa)$ [V1], we have

$$
\begin{aligned}
\int_{\gamma(z)} N(t, z)= & q(\kappa)^{-\frac{m_{2}}{4}+\frac{k(k-1)}{4}}\left(m_{2}\right)_{q(\kappa)} \ldots\left(m_{2}-k+1\right)_{q(\kappa)} \cdot u^{\left(\frac{m_{1} m_{2}}{2}-k\left(m_{1}+m_{2}\right)+k(k-1)\right) / k} \\
& \times k ! \int \prod_{j=1}^{k} x_{j}^{-m_{1} / k}\left(1-x_{j}\right)^{-m_{2} / \kappa} \prod_{1 \leqq j<l \leqq k}\left(x_{l}-x_{l}\right)^{2 / \kappa} A(x) d x_{1} \wedge \cdots \wedge d x_{k} \\
& \cdot f^{l} v_{m_{1}} \otimes f^{k-l} v_{m_{2}},
\end{aligned}
$$

and

$$
A_{l}(x)=\sum_{\sigma \in S(k ; l, k-l)} \prod_{i=1}^{k} \frac{1}{x_{l}-y_{\sigma(l)}},
$$

where $y_{1}=0, y_{2}=1$, and $S(k ; l, k-l)$ is defined in (3.2.2). 
The value of the integral over $\Delta$ is considered in the sense of analytic continuation from the domain of parameters where $\kappa$ is positive and $m_{1}, m_{2}$ are negative.

This formula is the decisive property of $\tilde{v}(z, \kappa)$ which allows us to prove (4.2.1.5) and (4.2.1.6).

Consider the single basic vector $v(c, 0, T, i d, \kappa) \in \mathscr{B}_{T, 1 \mathrm{~d}}(\kappa)$ of the one dimensional space $\operatorname{Sing} L_{\lambda}^{\text {id }}(q=q(\kappa))$. (4.4.6) implies that

$$
\int_{v(z, \kappa) v(c, 0, T, \text { id, } \kappa)} N=(2 \pi \kappa)^{-k / 2} q(\kappa)^{-\frac{m_{2}}{2}+\frac{3}{4} k(k-1)}
$$

$$
\begin{aligned}
& \times u^{\left(\frac{m_{1} m_{2}}{2}-k\left(m_{1}+m_{2}\right)+k(k-1)\right) / \kappa} k ! I_{k}\left(m_{1}, m_{2} ; \kappa\right) \\
& \times \prod_{j=0}^{k-1}\left(\left(1-\frac{\kappa}{m_{1}+m_{2}-2 k+j+2}\right)\left(m_{2}-j\right)_{q(\kappa)}\right) \cdot\left\{v_{1},{ }_{k} v_{2}\right\},
\end{aligned}
$$

where the vector $\left\{v_{1}, k_{k} v_{2}\right\} \in \operatorname{Sing}\left(L\left(m_{1}\right) \otimes L\left(m_{2}\right)\right)_{\lambda}$ is given by (1.4.1) and $I_{k}\left(m_{1}, m_{2} ; \kappa\right)$ is the Selberg integral given by (1.5.1). By (1.5.2) and (1.5.3), we have

$$
\int_{v(z, \kappa) v(c, 0, T, 1 \mathrm{~d}, \kappa)} N=C(c, T, \kappa) \cdot u^{\left(\frac{m_{1} m_{2}}{2}-k\left(m_{1}+m_{2}\right)+k(k-1)\right) / \kappa}\left\{v_{1},{ }_{k} v_{2}\right\} .
$$

The right-hand side is exactly the asymptotic solution defined by (2.3.1). This proves (4.2.1.5).

Now assume that $\kappa=i s, s \in \mathbb{R}$, and $s \rightarrow+0$. We compute the asymptotic expansion of the right-hand side of (4.5.4) using (1.5.1) and the Stirling formula. This shows that the asymptotic expansion of $\pi_{\mathrm{dd}}(\kappa) v(c, 0, T, i d, \kappa)$ has the form $(2.5 .8)$ and the properties described in (2.5.7) and (2.5.9).

According to the construction in [V1], the class $v(z, \kappa) v(c, 0, T, i d, \kappa)$ is flat with respect to $\kappa$, see (3.4). The function $\Phi(t, z)$ has exactly one $S_{k}$-orbit of $t$-critical points, see (3.5). To show that the class sits on this orbit we need the following lemma.

(4.5.6). Lemma. Let $[\gamma(\kappa)] \in H_{k}\left(\mathscr{U}_{k, 2}(z), \mathscr{S}(\kappa)\right)$ be a class flat with respect to $\kappa$. Then $[\gamma(\kappa)]$ sits on the orbit of $t$-critical points of $\Phi(t, z)$ or for any $P>0$ there exist a chain

$$
\gamma(\kappa)=\sum_{j=1}^{M}\left(c_{j}, \alpha_{j}(\kappa)\right)
$$

representing $[\gamma(\kappa)]$, flat with respect to $\kappa$, and such that, for every $j=1, \ldots, m$, we have

$$
\lim _{\substack{k=l s \\ s \rightarrow+0}} q(\kappa)^{P} \sup _{c_{J}}\left|\alpha_{J}(\kappa)\right|=0 .
$$

(4.5.8). Corollary. If $[\gamma(\kappa)]$ does not sit on the $\mathscr{S}_{k}$ orbit then

$$
\lim _{\substack{\kappa=i s \\ s \rightarrow+0}} q(\kappa)^{P} \int_{[\gamma(\kappa)]} N=0
$$

for all $P$.

Proof. First, remark that $\Phi^{\kappa}$ is a rational function on $\mathscr{U}_{k, 2}(z)$. 
Now consider the compactification of $\mathscr{U}_{k, 2}(z)$ in $\left(\mathbb{C} P^{1}\right)^{k}$. Let $X \subset\left(\mathbb{C} P^{1}\right)^{k}$ be the divisor of singularities of $\Phi$. A resolution of singularities of $X \subset\left(\mathbb{C} P^{l}\right)^{k}$ is a proper analytic map $F:(A \subset B) \rightarrow\left(X \subset\left(\mathbb{C} P^{1}\right)^{k}\right)$ such that $A$ is a divisor with normal crossings in a nonsingular $B$ and $\left.F\right|_{B-A}: B-A \rightarrow\left(\mathbb{C} P^{1}\right)^{k}-X$ is a biholomorphism. The condition $k \leqq \min \left(m_{1}, m_{2}\right)$ implies that there exists a resolution having the following property:

For any point $a \in A$, there exists local coordinates $u_{1}, \ldots, u_{k}$ on $B$ centered at $a$ and such that $A$ is defined by equation

$$
u_{1} \cdot \cdots \cdot u_{r}=0
$$

for some $r \in\{1, \ldots, k\}$ and

$$
\Phi^{\kappa} \circ F(u)=u_{1}^{l_{1}} \cdot \cdots \cdot u_{r}^{l_{1}}
$$

where $l_{1} \cdot \cdots \cdot l_{r} \neq 0$.

Let $S=\kappa \ln \Phi$. Then $S=\sum_{j=1}^{r} l_{j}\left(\ln \left|u_{j}\right|+i \arg u_{j}\right)$. Introduce the new real coordinates $v_{j}=\ln \left|u_{j}\right|$ and $w_{j}=\arg u_{j}$. Let $H_{1}, \ldots, H_{r}$ be arbitrary real numbers such that $H_{1} l_{1}+\cdots+H_{r} l_{r}=-1$. Set $Y=\sum_{j} H_{j} \frac{\partial}{\partial w_{j}}$. The vector field $Y$ decreases $\operatorname{Im} S$ in a neighborhood of the point $a$.

To prove the lemma we start with an arbitrary representing chain $\gamma(\kappa)$, flat with respect to $\kappa$. Then we deform $\gamma(\kappa)$ into the direction of decrease of $\operatorname{Im} S$. To deform the chain we use local fields $\{Y\}$ constructed above in a neighborhood of $X$ and we use the field $\operatorname{grad}(\operatorname{Im} S)$ in the "finite" part of the $\left(\mathbb{C} P^{1}\right)^{k} \backslash X$. This procedure will push the chain onto the orbit of $t$-critical points or will create a new representing chain with property (4.5.7).

The lemma and Theorem (4.2.1) for $n=2$ are proved.

(4.6). Proof of Theorem (4.2.1). To simplify notation we will prove the theorem for the case $n=3, \sigma=i d \in S_{3}$, and for the 3-tree shown in (4.6.1). The general case is completely similar.

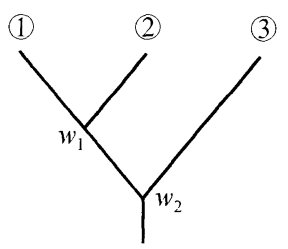

In this case the asymptotic zone has the form $u=u_{T \text {, id }}=\left\{z_{1}+z_{2}+z_{3}, u_{1}, u_{2}\right\}$, where $z_{3}-z_{2}=u_{2}, z_{2}-z_{1}=u_{1} u_{2}$. If $u_{1}$ and $u_{2}$ are small, then $z_{2}-z_{1} \ll z_{3}-z_{2}$.

Let the tops of $T$ be marked by nonnegative integers $m_{1}, m_{2}, m_{3}$ and let $c$ be an admissible coloring of $T$ having level $\lambda=m_{1}+m_{2}+m_{3}-2 k$. Set $c_{1}=c\left(w_{1}\right), c_{2}=$ $c\left(w_{2}\right)$, then $c_{1}+c_{2}=k$.

Let $v_{m}$ be the generating vector of the $s l_{2}$ module $L(m)$. Let $\bar{v}_{m}$ be the generating vector of the $U_{q=q(\kappa)}$ module $L(m, q=q(\kappa))$.

Set $v=\left\{\left\{v_{m_{1}}, c_{1} v_{m_{2}}\right\}, c_{2} v_{m_{3}}\right\}, \bar{v}=\left(\left(\bar{v}_{m_{1}}, c_{1} \bar{v}_{m_{2}}\right),{ }_{c_{2}} \bar{v}_{m_{3}}\right)$, see notations in (1.2.1) and (1.4.1)

Consider the solution to the $\mathrm{KZ}$ equation given by

$$
\psi: z \mapsto \int_{v(z, \kappa) \bar{v}} N,
$$


where $z=\left(z_{1}, z_{2}, z_{3}\right), z_{1}<z_{2}<z_{3}$. Our problem is to show the following statements (4.6.3) and (4.6.4):

$$
\begin{aligned}
\psi(z(u)) & =C(c, T, \kappa) \cdot u_{1}^{\mu_{1} / \kappa} u_{1}^{\mu_{2} / \kappa}(v+\mathcal{O}(u)), \\
C(c, T, \kappa) & =\frac{k !}{c_{1} ! c_{2} !} \cdot J_{c_{1}}\left(m_{1}, m_{2} ; \kappa\right) \cdot J_{c_{2}}\left(m^{1}, m_{3} ; \kappa\right),
\end{aligned}
$$

where $m^{1}=m_{1}+m_{2}-2 c_{1}$, see (1.5.3), and the real numbers $\mu_{1}, \mu_{2}$ are defined in (2.3.1).

(4.6.4). Theorem. Assume that $\kappa=i s, s \rightarrow+0$. Then for small $u_{1}, u_{2}$ the class $v(z, \kappa) \bar{v}$ sits on the orbit of the $t$-critical point $\left(t^{c}(z), z\right)$ such that

$$
\begin{aligned}
t_{p}^{c}(z)-z_{1} & =\left(a_{p}+\mathcal{O}(u)\right) u_{1} u_{2}, & & p=1, \ldots, c_{1}, \\
t_{p+c_{1}}^{c}(z)-z_{2} & =\left(b_{1}+\mathcal{O}(u)\right) u_{2}, & & p=1, \ldots, c_{2},
\end{aligned}
$$

where $\left(a_{1}, \ldots, a_{c_{1}}\right)$ (resp. $\left.\left(b_{1}, \ldots, b_{c_{2}}\right)\right)$ is a critical point of $\Phi_{c_{1}, m_{1}, m_{2}, k}$ (resp. $\left.\Phi_{c_{2}, m^{1}, m_{3}, \kappa}\right)$, see (3.6.2). Moreover, the asymptotic expansion of $\psi(z(u))$ as $s \rightarrow$ +0 has the form described in (2.5.6) and the properties described in (2.5.7) and (2.5.9).

Proof. To prove these statements we will choose a special chain representation for the class $v(z, \kappa) \bar{v}$. We will use the construction of iterated cycles described in [V1, Sect. 14].

Consider the space $\mathbb{C}^{2+c_{1}}$ with coordinates $z_{1}, z_{2}, t_{1}, \ldots, t_{c_{1}}$. Let

$$
\begin{gathered}
p r_{I}: \mathbb{C}^{2+c_{1}} \rightarrow \mathbb{C}^{2},\left(z_{1}, z_{2}, t\right) \mapsto\left(z_{1}, z_{2}\right), \\
\Phi_{I}=\left(z_{2}-z_{1}\right)^{\frac{m_{1} m_{2}}{2 \kappa}} \prod_{1 \leqq l<J \leqq c_{1}}\left(t_{l}-t_{J}\right)^{2 / \kappa} \cdot \prod_{l=1}^{c_{1}} \prod_{j=1}^{2}\left(z_{J}-t_{l}\right)^{-m_{j} / \kappa} .
\end{gathered}
$$

By (4.4.6) we have a map

$$
\begin{aligned}
v_{I}\left(z_{1}, z_{2}, \kappa\right): \operatorname{Sing}\left(L \left(m_{1}, q\right.\right. & \left.=q(\kappa)) \otimes L\left(m_{2}, q=q(\kappa)\right)\right)_{m^{1}=m_{1}+m_{2}-2 c_{1}} \\
& \longrightarrow H_{c_{1}}\left(p r_{I}\left(z_{1}, z_{2}\right)^{-1}, \mathscr{S}_{I}(\kappa)\right)_{-},
\end{aligned}
$$

where $z_{1}, z_{2} \in \mathbb{R}, z_{1}<z_{2}$, and $\mathscr{S}_{I}(\kappa)$ is the local system defined by $\Phi_{I}$.

For $z_{1}=0, z_{2}=1$, fix a chain representative $\gamma_{I}$ for the class $\left[\gamma_{I}(0,1)\right]=$ $v_{I}(0,1, \kappa)\left(\bar{v}_{m_{1}}, c_{1} \bar{v}_{m_{2}}\right)$, flat with respect to $\kappa$, cf. (3.4.1).

For fixed numbers $z_{1}^{0}, z_{2}^{0}$ the map

$$
T:\left(z_{1}, z_{2}, t_{1}, \ldots, t_{c_{1}}\right) \mapsto\left(\left(z_{2}^{0}-z_{1}^{0}\right) z_{1}+z_{1}^{0},\left(z_{2}^{0}-z_{1}^{0}\right) z_{2}+z_{1}^{0},\left(z_{2}^{0}-z_{1}^{0}\right) t_{1}+z_{1}^{0}, \ldots\right)
$$

sends $\operatorname{pr}_{I}^{-1}(0,1)$ to $p_{I}^{-1}\left(z_{1}^{0}, z_{2}^{0}\right)$. Hence, the image of $\gamma_{I}$ under $T$ gives a representative

$$
\gamma_{I}\left(z_{1}^{0}, z_{2}^{0}\right)=\sum_{j=1}^{M_{I}}\left(C_{j}^{I}\left(z_{1}^{0}, z_{2}^{0}\right), \alpha_{j}^{I}(\kappa)\right)
$$

of the class $v_{I}\left(z_{1}^{0}, z_{2}^{0}, \kappa\right)\left(\bar{v}_{m_{1}}, c_{1} \bar{v}_{m_{2}}\right)$ for arbitrary $z_{1}^{0}<z_{2}^{0}$. This representation is flat with respect to $\kappa$. 
Similarly, consider the space $\mathbb{C}^{2+c_{2}}$ with coordinates $z_{2}, z_{3}, t_{c_{1}+1}, \ldots, t_{k}$. Let

$$
\begin{gathered}
p r_{I I}: \mathbb{C}^{2+c_{2}} \rightarrow \mathbb{C}^{2}, \quad\left(z_{2}, z_{3}, t\right) \mapsto\left(z_{2}, z_{3}\right), \\
\Phi_{I I}=\left(z_{3}-z_{2}\right)^{m^{1} m_{3} / 2 \kappa} \prod_{c_{1}<l<j \leqq k}\left(t_{l}-t_{j}\right)^{2 / \kappa} \prod_{l=c_{1}+1}^{k}\left(z_{2}-t_{l}\right)^{-m^{1} / \kappa}\left(z_{3}-t_{l}\right)^{-m_{3} / \kappa} .
\end{gathered}
$$

By (4.4.6) we have a map

$$
\begin{gathered}
v_{I I}\left(z_{2}, z_{3}, \kappa\right): \operatorname{Sing}\left(L\left(m^{1}, q=q(\kappa)\right) \otimes L\left(m_{3}, q=q(\kappa)\right)_{m^{1}+m_{3}-2 c_{2}}\right. \\
\rightarrow H_{c_{2}}\left(\operatorname{pr}_{I I}^{-1}\left(z_{2}, z_{3}\right), \mathscr{S}_{I I}(\kappa)\right)_{-},
\end{gathered}
$$

where $z_{2}, z_{3} \in \mathbb{R}, z_{2}<z_{3}$, and $\mathscr{S}_{I I}(\kappa)$ is defined by $\Phi_{I I}$. As above, construct a chain representation

$$
\gamma_{I I}\left(z_{2}, z_{3}\right)=\sum_{l=1}^{M_{I I}}\left(C_{l}^{I I}\left(z_{2}, z_{3}\right), \alpha_{l}^{I l}(\kappa)\right)
$$

of the class $v_{I I}\left(z_{2}, z_{3}\right)\left(\bar{v}_{m^{1}}, c_{2} \bar{v}_{m_{3}}\right)$ which is flat with respect to $\kappa$.

Using the chains $\gamma_{I}, \gamma_{I I}$, we will construct a chain representing $v(z, \kappa)$ $\left(\left(\bar{v}_{m_{1}}, c_{1} \bar{v}_{m_{2}}\right),{ }_{c_{2}} \bar{v}_{m_{3}}\right)$.

Let $u_{1}, u_{2}$ be small, then $z_{2}-z_{1} \ll z_{3}-z_{2}$.

Identify $\mathrm{pr}_{I}^{-1}\left(z_{1}, z_{2}\right)$ with $\mathbb{C}^{c_{1}}$ using the coordinates $t_{1}, \ldots, t_{c_{1}}$, identify $\operatorname{pr}_{I I}^{-1}\left(z_{2}, z_{3}\right)$ with $\mathbb{C}^{c_{2}}$ using the coordinates $t_{c_{1}+1}, \ldots, t_{k}$, and identify $\operatorname{pr}_{3, k}^{-1}\left(z_{1}, z_{2}, z_{3}\right)$ with $\mathbb{C}^{k}=\mathbb{C}^{c_{1}} \times \mathbb{C}^{c_{2}}$.

Using these identifications, for any $j=1, \ldots, M_{I}, l=1, \ldots, M_{I I}$, define a $k$-cell $C_{j l}\left(z_{1}, z_{2}, z_{3}\right)$ in $p r_{3, k}^{-1}\left(z_{1}, z_{2}, z_{3}\right)$ :

$$
C_{j l}\left(z_{1}, z_{2}, z_{3}\right)=C_{j}\left(z_{1}, z_{2}\right) \times C_{l}\left(z_{2}, z_{3}\right) .
$$

Using $\alpha_{J}^{I I}$ and $\alpha_{l}^{I I}$ we will define a coefficient of $C_{j l}$ in the local system $\mathscr{S}(\kappa)$ defined by the function $\Phi$ described in (3.1.1) for $n=3$.

$\alpha_{j}^{I}$ has the form $\varepsilon_{j}^{I} \cdot \beta_{j}^{I}$, where $\varepsilon_{j}^{I}$ is a rational function of $q(\kappa)^{\frac{1}{4}}$ and $\beta_{j}^{I}$ is a branch of $\Phi_{I}$ over $C_{j}^{I}$. The coefficient $\alpha_{l}^{I I}$ has the form $\varepsilon_{l}^{I I} \cdot \beta_{l}^{I I}$, where $\varepsilon_{l}^{I I}$ is a rational function of $q(\kappa)^{\frac{1}{4}}$ and $\beta_{l}^{I I}$ is a branch of $\Phi_{I I}$ over $C_{l}^{I I}$. We define the coefficient of $C_{j l}$ in $\mathscr{S}(\kappa)$ by

$$
\alpha_{j l}=\varepsilon_{j}^{I} \cdot \varepsilon_{l}^{I I} \cdot \beta_{i j}
$$

where $\beta_{i j}$ is the branch of $\Phi$ over $C_{j l}$ defined below.

Any branch of $\Phi_{I}, \Phi_{I I}$, or $\Phi$ is defined by determining arguments of all differences $t_{l}-t_{j}, z_{j}-t_{l}, z_{l}-z_{j}$ in the formulae for $\Phi_{I}, \Phi_{I I}$, and $\Phi$. Therefore, we assume that over $C_{j}^{I}$ (resp. $C_{l}^{I I}$ ) the argument of every difference in $\Phi_{I}$ (resp. $\Phi_{I I}$ ) is fixed. Let us determine the argument of every difference in $\Phi$ by the following rule:

Choose an argument of $z_{3}-z_{2}, z_{2}-z_{1}, z_{b}-t_{a}$ (for $a>c_{1}, b=2,3$ ), $z_{b}-t_{a}$ (for $a<c_{1}$ and $b=1,2$ ), $t_{a}-t_{b}$ (for $1 \leqq a<b \leqq c_{1}$ and for $c_{1}<a<b \leqq k$ ) the same as those in $\beta_{j}^{I}$ and $\beta_{l}^{I I}$. The function $z_{1}-t_{a}$ for $a>c_{1}$ is approximately equal to $z_{2}-t_{a}$ on $C_{j l}$. Choose the argument of $z_{1}-t_{a}$ which is close to the argument of $z_{2}-t_{a}$ in $\beta_{l}^{I I}$.

The function $z_{3}-t_{a}$ for $a \leqq c_{1}$ is approximately equal to $z_{3}-z_{2}$ on $C_{j l}$. Choose the argument of $z_{3}-t_{a}$ which is close to the argument of $z_{3}-z_{2}$ chosen in $\beta_{l}^{I I}$. 
The function $t_{a}-t_{b}$ for $a \leqq c_{1}$ and $b>c_{1}$ is approximately equal to $z_{2}-t_{b}$ on $C_{j l}$. Choose the argument of $t_{a}-t_{b}$ which is close to the argument of $z_{2}-t_{b}$ on $C_{j l}$.

It is easy to see that

$$
\tau\left(z_{1} z_{2} z_{3}\right)=\sum_{j}^{M_{I}} \sum_{l}^{M_{I I}}\left(C_{J l}, \alpha_{j l}\right)
$$

is a cycle. The group $S_{k}$ acts on the space of chains with coefficients in $\mathscr{S}(\kappa)$. Set

$$
\gamma\left(z_{1}, z_{2}, z_{3}\right)=\frac{1}{c_{1} ! c_{2} !} \sum_{\chi \in S_{k}}(-1)^{|\chi|} \chi \cdot \tau\left(z_{1}, z_{2}, z_{3}\right) .
$$

According to [V1, Sect. 14] we have

The chain $\gamma\left(z_{1}, z_{2}, z_{3}\right)$ represents the class $v\left(z_{1}, z_{2}, z_{3}\right) \bar{v}$.

Now introduce the new coordinates $x_{1}, \ldots, x_{k}$ in $\operatorname{pr}_{3, k}^{-1}\left(z_{1}, z_{2}, z_{3}\right)$ :

$$
\begin{array}{ll}
t_{p}-z_{1}=x_{p} u_{1} u_{2}, & p=1, \ldots, c_{1}, \\
t_{p}-z_{2}=x_{p} u_{2}, & p=c_{1}+1, \ldots, k .
\end{array}
$$

The cells $C_{j l}$ of the chain $\tau$ written in coordinates $x_{1}, \ldots, x_{p}$ do not depend on $u_{1}, u_{2}$. The dependence of $\Phi$ in those coordinates has the form

$$
\Phi=u_{1}^{\mu_{1} / \kappa} u_{2}^{\mu_{2} / \kappa}\left(\Phi_{c_{1}, m_{1}, m_{2}, \kappa}\left(x_{1}, \ldots, x_{c_{1}}\right) \cdot \Phi_{c_{2}, m^{1}, m_{3}, \kappa}\left(x_{c_{1}+1}, \ldots, x_{k}\right)+\mathcal{O}(u)\right) .
$$

Knowing that $\gamma_{I}(0,1)$ sits on $\left(a_{1}, \ldots, a_{c_{1}}\right)$ and $\gamma_{I I}(0,1)$ sits on $\left(b_{1}, \ldots, b_{c_{2}}\right)$, we may conclude that $\gamma$ sits on the $t$-critical point described in (4.6.4). Knowing that integrals over $\gamma_{I}$ and $\gamma_{I I}$ have the desired asymptotic expansion, see (4.5), we may conclude that the asymptotic expansion of the function $\psi$ has the form described in (2.5.6) and the properties described in (2.5.7). Formula (4.6.9) also shows that the integral

$$
\int_{\gamma\left(z_{1}, z_{2}, z_{3}\right)} N
$$

is the asymptotic solution $\psi_{c, T \text {, id }}$

Theorem (4.2.1) is proved.

\section{References}

[AGV] Arnold, V.I., Gusein-Zade, S.M., Varchenko, A.N.: Singularities of Differentiable Maps, 2, Birkhauser, 1988

[B] Bethe, H.: Z. Phys. 71, 205 (1931)

[Ba] Babujian, H.M.: Off-shell Bethe ansatz equation and N-point correlators in the $S U(2)$ WZNW theory. J. Phys. A: Math. Gen. 26, 6981-6990 (1993)

[BF] Babujian, H.M., Flume, R.: Off shell Bethe ansatz equation for Gaudin magnets and solutions of Knizhnik-Zamolodchikov equations. Preprint Bonn-HE-93-30

[D] Drinfeld, V.: Quasi-Hopf algebras. Leningrad Math. J. 1, no. 6 (1989)

[FFR] Feigin, B., Frenkel, E., Reshetikhin, N.: Gaudin model, Bethe ansatz and correlation functions at the critical level. Preprint, 1994 
[FT] Faddeev, L., Takhtajan, L.: Uspehi Mat. Nauk 34, no. 5, 13-63 (1979)

[FW] Felder, G., Wieczerkowski, C.: Topological representations of $U_{q}\left(s l_{2}\right)$. Commun. Math. Phys. 138, 583-605 (1991)

[G] Gaudin, M.: Diagonalizations d'une classe d'hamiltoniens de spin. Jour. de Physique 37, no. 10, 1087-1098 (1976)

[K] Kashiwara, M.: Crystallizing the $q$-Analogue of Universal Enveloping Algebras. Commun. Math. Phys. 133, 249-260 (1990)

[Ko] Kohno, T.: Monodromy representations of braid groups and Yang-Baxter equations. Ann. Inst. Fourier 37, 139-160 (1987)

[KR] Kirillov, A., Reshetikhin, N.: Representations of the algebra $U_{q}(s l(2))$, $q$-orthogonal polynomials and invariants of links. Preprint, Leningrad, 1988

[KZ] Knizhnik, V., Zamolodchikov, A.: Current algebra and Wess-Zumino models in two dimensions. Nucl. Phys. B 247, 83-103 (1984)

[M] Mehta, M.: Random Matrices. New York: Acad. Press, 1991

[R] Reshetikhin, N.: Jackson type integrals, Bethe vectors, and solutions to a difference analog of the Knizhnik-Zamolodchikov system, Lett. Math. Phys. 26, 153-165 (1992)

[RV] Reshetikhin, N., Varchenko, A.: Quasiclassical Asymptotics of Solutions to the KZ Equations. Preprint, February, 1994

[Sz] Szego, G.: Orthogonal Polynomials. Providence, RI: AMS, 1939

[TV] Tarasov, V., Varchenko, A.: Jackson integral representations for solutions of the KZ equation, St. Petersburg Math. J. 6, no. 2 (1994)

[SV] Schechtman, V., Varchenko, A.: Arrangements of hyperplanes and Lie algebra homology. Invent. Math. 106, 139-191 (1991)

[V1] Varchenko, A.: Multidimensional hypergeometric functions and representation theory of Lie algebras and quantum groups. Singapore: World Scientific, Advanced Series in Mathematical Physics, vol. 21, 1995

[V2] Varchenko, A.: Critical points of the product of powers of linear functions and families of bases of singular vectors. Compositio Math., to appear

Communicated by G. Felder 
